\title{
K-DIFFERENCED VECTOR RANDOM FIELDS
}

\author{
A Dissertation by \\ Rehab Alsultan \\ Bachelor of Science, King Faisal University, 2005 \\ Master of Science, Pittsburg State University , 2010
}

Submitted to the Department of Mathematics, Statistics, and Physics and the faculty of the Graduate School of

Wichita State University

in partial fulfillment of

the requirements for the degree of

Doctor of Philosophy

May 2015 
(C) Copyright 2015 by Rehab Alsultan

All Rights Reserved 


\section{K-DIFFERENCED VECTOR RANDOM FIELDS}

The following faculty members have examined the final copy of this dissertation for form and content, and recommend that it be accepted in partial fulfillment of the requirement for the degree of Doctor of Philosophy with a major in Applied Mathematics.

Dr Chunsheng Ma, Committee Chair

Dr Kirk Lancaster, Committee Member

Dr Hari Mukerjee, Committee Member

Dr Tianshi Lu, Committee Member

Dr Yanwu Ding, Outside Committee Member

Accepted for the College of Liberal Arts and Sciences

Ron Matson, Dean

Accepted for the Graduate School

Abu S.M. Masud, Interim Dean 


\section{DEDICATION}

To my parents 


\section{ACKNOWLEDGEMENTS}

I would first like to acknowledge God and then, secondly, all my family who have supported me and given me strength to come to this point. This dissertation would not have been possible without the guidance and help of several individuals who, in one way or another, have contributed and extended their valuable assistance in the preparation and completion of this study. My first debt of gratitude goes to my advisor, Professor Chunseng Ma, who has truly been an inspiration. Without his invaluable guidance, this dissertation would not have been possible. I would also like to express my gratitude to all the members of my committee for the contribution of their valuable time. 


\begin{abstract}
There is a great demand for analyzing multivariate measurements observed across space and over time, due to an increasing wealth of multivariate spatial, temporal, or spatiotemporal data, which may be treated as the realizations of vector (multivariate) random fields. As one of the most important random fields in theory and application, Gaussian random field has been extensively investigated in the literature. Non-Gaussian models and random fields are often encountered in many natural and applied science areas, with specific reasons for assuming particular non-Gaussian finite-dimensional distributions in practice.
\end{abstract}

One of the objectives of this dissertation is to introduce a new non-Gaussian vector random field, which belongs to the family of elliptically contoured vector random fields. This new field is named the K-differenced vector random fields, because its finite-dimensional densities are the difference of two Bessel K functions. A K-differenced vector random field is of second-order and allows for any possible correlation structure, just as a Gaussian one does. It includes a Laplace vector random field as a limiting case. This dissertation studies the properties of the K-differenced vector random field and proposes some covariance matrix structures for not only a K-differenced vector random field but also a second-order elliptically contoured one.

Other objectives of this dissertation are to construct the K-differenced random variable or random vector as the scale mixture of normal random variables or vectors and to derive its density and characteristic functions. Simulations of the K-differenced distribution have been made through Monte Carlo procedures. Maximum likelihood estimators of the parameters for the simulations are found numerically via MatLab. 


\section{TABLE OF CONTENTS}

\section{Chapter}

1 Introduction . . . . . . . . . . . . . . . . . . . . . 1

2 K-differenced Random Variable . . . . . . . . . . . . . . . . . . . . . . . . 6

2.1 Stochastic representation . . . . . . . . . . . . . . . . . 6

2.2 Density function . . . . . . . . . . . . . . . . . . . . . . . . . . . . . . . . .

2.3 Characteristic function . . . . . . . . . . . . . . . . . 11

2.4 Simulation . . . . . . . . . . . . . . . . . . . . . . . . . . . . . . . .

2.5 Maximum likelihood estimation . . . . . . . . . . . . . . 15

3 K-differenced Random Vector . . . . . . . . . . . . . . . . . . . 22

3.1 Stochastic representation . . . . . . . . . . . . . . . . . . . . 22

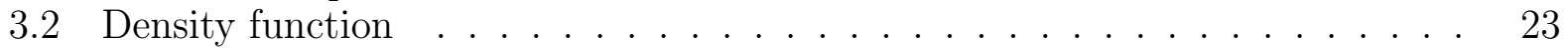

3.3 Characteristic function . . . . . . . . . . . . . . 26

3.4 Simulation . . . . . . . . . . . . . . . . . . . 27

3.5 Maximum likelihood estimation . . . . . . . . . . . . . . . . . . 29

4 K-differenced Vector Random fields . . . . . . . . . . . . . . . . . . . . 35

4.1 Stochastic Construction . . . . . . . . . . . . . . . . 35

4.2 Finite-dimensional characteristic functions . . . . . . . . . . . . . . 36

4.3 Properties . . . . . . . . . . . . . . . . . . . . 37

5 Some Covariance Matrix Structures . . . . . . . . . . . . . . . . . . . . . . . . 40

5.1 Some notations and facts . . . . . . . . . . . . . . . . . . . . 40

5.2 Covariance matrix structure I . . . . . . . . . . . . . . . . . . 42

5.3 Covariance matrix structure II . . . . . . . . . . . . . . . . . . . 48

6 Concluding Remarks . . . . . . . . . . . . . . . . . . 55

REFERENCES . . . . . . . . . . . . . . . . . . . 57

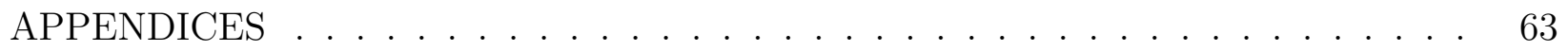

A MatLab code used in the K-differenced random variable . . . . . . . . . . 64

B MatLab code used in the K-differenced random vector . . . . . . . . . . . 69 


\section{CHAPTER 1}

\section{Introduction}

The major aim of this thesis is to construct or build a new type of non-Gaussian vector random fields, which is an elliptically contoutred vector random field and is named a K-differenced vector random field, and to investigate its properties.

Since the turn of the 20th century, there are numerous approaches of constructing symmetric multivariate distributions. In the survey paper, multivariate distributions at a crossroad, written by Kotz (1975) and in the joint paper of Goodman and Kotz (1981), Samuel Kotz presented a more or less systematic classification of theses methods of extension of multivariate distributions based on various criteria such as the type of dependence, analogy of mathematical form, model and characterizations. Although these problems have not been completely and fully solved, substantial progress has been made in the area. Researchers in statistical distributions have succeeded in developing, in several decades, and shifting the centre of gravity of univariate distributions from the normal distribution. By this success, researchers have more recently turned their attention and methodology to analysis of multivariate distributions other than the normal one. In this research, a class of spherically and elliptically contoured distributions which are extensions of multivariate normal distributions are defined and have been studied. Here we concentrate on the mixture of normal distributions which is the most important class of elliptically symmetric distributions (see Fang, K. et al. (1990), p.1).

Although, as indicated in an excellent review of elliptically symmetric distributions by Chmielewski (1981), earlier papers on this topic can be traced to Maxwell (1860), Bartlett (1934) and Hartman and Wintner (1940), the modern era of research in this field starts with the engineering applications considered by Blake and Thomas (1968) and McGraw and Wag- 
ner (1968), followed by Chu (1973), and more prominently for statisticians with Dempster (1969) and the first organized presentation of this family by Kelker (1970). Since then we are witnessing a substantial well-organized and ever increasing amount of research of properties and applications of these distributions (see Fang, K. et al. (1990), p. 26).

There are several ways to define spherically and elliptically contoured distributions. A spherical distribution is an extension of the standard multinormal distribution and an elliptical contoured distribution is an extension of the multivariate normal distribution. It is well known that the an n-variate normal random vector $\mathbf{Z}$ with mean $\boldsymbol{\mu}$ and variance-covariance matrix $\boldsymbol{\Sigma}$ can be defined as

$$
\mathbf{Z} \stackrel{d}{=} \boldsymbol{A}^{\prime}\left(\begin{array}{c}
Y_{1} \\
Y_{2} \\
\vdots \\
Y_{n}
\end{array}\right)+\boldsymbol{\mu}
$$

where $Y_{1}, \ldots, Y_{n}$ are independent standard normal random variables, $\boldsymbol{\Sigma}=\mathbf{A}^{\prime} \mathbf{A}$, and $\stackrel{d}{=}$ means that both sides have the same distributions. The characteristic function of $\mathbf{Z}$ has the form

$$
E e^{i \mathbf{Z}^{\prime} \boldsymbol{\omega}}=e^{i \boldsymbol{\omega}^{\prime} \boldsymbol{\mu}-\frac{1}{2} \boldsymbol{\omega}^{\prime} \boldsymbol{\Sigma} \boldsymbol{\omega}}, \quad \boldsymbol{\omega} \in \mathbb{R}^{n}
$$

where $i$ is the imaginary unit.

An $n$-variate random vector $\mathbf{Z}$ is said to follow an elliptically contoured distribution, if its characteristic function is of the form

$$
E e^{i \mathbf{Z}^{\prime} \boldsymbol{\omega}}=e^{i \boldsymbol{\omega}^{\prime} \mu} \Psi\left(\boldsymbol{\omega}^{\prime} \boldsymbol{\Sigma} \boldsymbol{\omega}\right), \quad \boldsymbol{\omega} \in \mathbb{R}^{n}
$$

for some scalar function $\Psi(\cdot)$, and its density, if exists, has the form 


$$
g\left((\mathbf{x}-\boldsymbol{\mu})^{\prime} \mathbf{\Sigma}^{-1}(\mathbf{x}-\boldsymbol{\mu})\right)
$$

for some nonnegative scalar function $g(\cdot)$. Thus, for elliptically contoured distributions, the contours of equal density are elliptical in shape.

Suppose that $\left\{\mathbf{Z}(x)=\left(Z_{1}(x), \ldots, Z_{m}(x)\right)^{\prime}, x \in \mathbb{D}\right\}$ is an m-variate stochastic process or random field, which is a set of m-variate random vectors on the same probability space, where the index set $\mathbb{D}$ could be a spatial, temporal, or spatio-temporal domain. If the variance of $Z_{k}(x)$ exists for every $x \in \mathbb{D}$ and $k=1, \ldots, m$, then this vector random field is said to be of second order. In this case the mean and covariance matrix functions of $\left\{\mathbf{Z}(x), x \in \mathbb{D}^{\prime}\right\}$ are defined by

$$
E \mathbf{Z}(x)=\left(Z_{1}(x), \ldots, Z_{m}(x)\right)^{\prime}, \quad x \in \mathbb{D},
$$

and

$$
\mathbf{C}\left(x_{1}, x_{2}\right)=E\left\{\left[\mathbf{Z}\left(x_{1}\right)-E \mathbf{Z}\left(x_{1}\right)\right]\left[\mathbf{Z}\left(x_{2}\right)-E \mathbf{Z}\left(x_{2}\right)\right]^{\prime}\right\}, \quad x_{1}, x_{2} \in \mathbb{D}
$$

respectively.

A diagonal entry of $\mathbf{C}\left(x_{1}, x_{2}\right), \mathbf{C}_{i i}\left(x_{1}, x_{2}\right)$, is called a direct covariance function, and an off-diagonal entry, $\mathbf{C}_{i j}\left(x_{1}, x_{2}\right), i \neq j$, is called a cross covariance function, $i, j=1, \ldots, m$. Also, $\{\mathbf{Z}(x), x \in \mathbb{D}\}$ is said to be stationary or homogeneous if

(i) the mean function $E \mathbf{Z}(x)$ does not depend on $x \in \mathbb{D}$; in other words, the mean function is a constant vector,

(ii) the covariance matrix function $\mathbf{C}\left(x_{1}, x_{2}\right)$ depends only on the lag $x_{1}-x_{2}$. 
In such case, we write $\mathbf{C}\left(x_{1}, x_{2}\right)$ as $\mathbf{C}\left(x_{1}-x_{2}\right)$ for simplicity.

There are two fundamental properties for a covariance matrix function $\mathbf{C}\left(x_{1}, x_{2}\right)$ :

(i) the transpose of $\mathbf{C}\left(x_{1}, x_{2}\right)$ equals $\mathbf{C}\left(x_{2}, x_{1}\right)$,

$$
\left(\mathbf{C}\left(x_{1}, x_{2}\right)\right)^{\prime}=\mathbf{C}\left(x_{2}, x_{1}\right) ;
$$

(ii) the inequality

$$
\sum_{i=1}^{n} \sum_{j=1}^{n} \mathbf{a}_{i}^{\prime} \mathbf{C}\left(x_{i}, x_{j}\right) \mathbf{a}_{j} \geq 0
$$

holds for every natural number $n$, any $x_{i} \in \mathbb{D}$, and any $\mathbf{a}_{i} \in \mathbb{R}^{m}, i=1, \ldots, n$.

Furthermore, for a given $m \times m$ matrix function $\mathbf{C}\left(x_{1}, x_{2}\right)$ on $\mathbb{D}$ that satisfies the above properties (i) and (ii), we can construct an m-variate Gaussian or elliptically contoured (spherically invariant) random field with mean $\mathbf{0}$ and $\mathbf{C}\left(x_{1}, x_{2}\right)$ as its covariance matrix function; see Ma (2011a).

Gaussian random field may be too limited to fit all data. Thus, we need non-Gaussian random fields. Non-gaussian models are encountered in many natural and applied science fields, for example finance (distribution of log-returns of a commodity), speech recognition (input distribution), and ocean engineering (distribution of navigation error). A question arises: how could we construct a non-Gaussian random field which is specially an elliptically contoured random field? A novel and simple way is introduced in Ma (2009) for constructing many such non-Gaussian random fields, with the corresponding finite-dimensional distribution identified. He derives such a field as a scale mixture of Gaussian vector random fields, 
while ensuring the existence of the second moments. Example of elliptically contoured random fields include Student's t, logistic, hyperbolic, Mittag-Leffler, and Linnik. Our goals of this dissertation are to construct a K-Differenced vector random field, and to investigate its properties.

In this dissertation we develop a new random field and call it the K-differenced vector random field, which is one of the elliptically contoured (spherically invariant) random fields. The rest of the thesis is organized as follows. A K-differenced random variable is introduced in Chapter 2, with density and characteristic functions derived. Simulation and maximum likelihood estimation of the K-differenced distribution also have been conducted. Chapter 3 discusses a natural extension of the K-differenced random variable to the random vector setting. Stochastic construction of the K-differenced vector random field is introduced in Chapter 4. Finally, in Chapter 5, we construct different types of covariance matrix structures that work for any second-order elliptically contoured vector random field including the K-differenced one. 


\section{CHAPTER 2}

\section{K-differenced Random Variable}

In this chapter we introduce the K-differenced distribution and study its properties. A K-differenced random variable is defined in Section 2.1 through a stochastic representation, and its density function and characteristic function are derived in Sections 2.2 and 2.3, respectively. Simulations are made in Section 2.4 using one of Monte Carlo methods, and the MatLab codes are put in Appendix A. Maximum likelihood estimations are found numerically in Section 2.5.

\subsection{Stochastic representation}

This section constructs a K-differenced random variable via a stochastic representation, and evaluates its moments.

To define a K-differenced random variable, we need a nonnegative random variable, U, say, which possesses the density function

$$
f_{U}(u)= \begin{cases}\frac{2\left(\exp \left(-\alpha_{2} u^{2}\right)-\exp \left(-\alpha_{1} u^{2}\right)\right)}{\left(\ln \alpha_{1}-\ln \alpha_{2}\right) u}, & u>0 \\ 0, & u \leq 0\end{cases}
$$

where $\alpha_{1}, \alpha_{2}$ are distinct constants, with $0<\alpha_{1}<\alpha_{2}$. A plot of density (2.1) is in in Figure 2.1.

The mean of the random variable $U$ is

$$
E U=2 \int_{0}^{\infty} \frac{u\left(\exp \left(-\alpha_{2} u^{2}\right)-\exp \left(-\alpha_{1} u^{2}\right)\right)}{\left(\ln \alpha_{1}-\ln \alpha_{2}\right) u} d u
$$




$$
\begin{aligned}
& =2 \int_{0}^{\infty} \frac{\left(\exp \left(-\alpha_{2} u^{2}\right)-\exp \left(-\alpha_{1} u^{2}\right)\right)}{\left(\ln \alpha_{1}-\ln \alpha_{2}\right)} d u \\
& =\frac{4}{2\left(\ln \alpha_{1}-\ln \alpha_{2}\right)} \int_{-\infty}^{\infty}\left\{\exp \left(-\alpha_{2} u^{2}\right)-\exp \left(\alpha_{1} u^{2}\right)\right\} d u \\
& =\frac{(2 \pi)^{1 / 2}}{2\left(\ln \alpha_{1}-\ln \alpha_{2}\right)}\left\{\frac{4}{\left(2 \alpha_{2}\right)^{1 / 2}} \int_{-\infty}^{\infty} \frac{1}{(2 \pi)^{1 / 2} \frac{1}{\left(2 \alpha_{2}\right)^{1 / 2}}} \exp \left(-\frac{u^{2}}{\frac{2}{2 \alpha_{2}}}\right) d u\right. \\
& =\frac{\left.-\frac{4}{\left(2 \alpha_{1}\right)^{1 / 2}} \int_{-\infty}^{\infty} \frac{1}{(2 \pi)^{1 / 2} \frac{1}{\left(2 \alpha_{1}\right)^{1 / 2}}} \exp \left(-\frac{u^{2}}{\frac{2}{2 \alpha_{1}}}\right)\right\} d u}{2\left(\ln \alpha_{1}-\ln \alpha_{2}\right)}\left\{\left(\alpha_{2}\right)^{-1 / 2}-\left(\alpha_{1}\right)^{-1 / 2}\right\}
\end{aligned}
$$

where the two integrals in the fourth equality are equal to 1, since they are integrals of the two normal density functions over the whole real line.

The second moment of the random variable $U$ is

$$
\begin{aligned}
& E U^{2}=2 \int_{0}^{\infty} \frac{u^{2}\left(\exp \left(-\alpha_{2} u^{2}\right)-\exp \left(-\alpha_{1} u^{2}\right)\right)}{\left(\ln \alpha_{1}-\ln \alpha_{2}\right) u} d u \\
& =\frac{2}{\left(\ln \alpha_{1}-\ln \alpha_{2}\right)}\left(\frac{1}{2} e^{-\alpha_{2} u^{2}}-\frac{1}{2} e^{-\alpha_{1} u^{2}}\right)_{0}^{\infty} \\
& =\left(\ln \alpha_{1}-\ln \alpha_{2}\right)^{-1}\left(\alpha_{2}^{-1}-\alpha_{1}^{-1}\right),
\end{aligned}
$$

and its variance is

$$
\begin{aligned}
& \operatorname{Var}(U)=E U^{2}-(E U)^{2} \\
& \quad=\left(\ln \alpha_{1}-\ln \alpha_{2}\right)^{-1}\left(\alpha_{2}^{-1}-\alpha_{1}^{-1}\right)-\frac{\pi}{4\left(\ln \alpha_{1}-\ln \alpha_{2}\right)^{2}}\left\{\left(\alpha_{2}\right)^{-1 / 2}-\left(\alpha_{1}\right)^{-1 / 2}\right\}^{2} .
\end{aligned}
$$


One extreme case of (2.1) is worth a mention here. When $\alpha_{1}$ tends to $\alpha_{2}$,

$$
\begin{gathered}
\lim _{\alpha_{1} \rightarrow \alpha_{2-}} \frac{2\left(\exp \left(-\alpha_{2} u^{2}\right)-\exp \left(-\alpha_{1} u^{2}\right)\right)}{\left(\ln \alpha_{1}-\ln \alpha_{2}\right) u} \\
=2 \alpha_{2} u \exp \left(-\alpha_{2} u^{2}\right), u>0,
\end{gathered}
$$

is the density function of a Weibull random variable.

A K-differenced random variable $\mathrm{Z}$ is formulated as

$$
Z=\lambda U Z_{0}+\mu
$$

where $\mu$ is constant, $Z_{0}$ is a normal (Gaussian) random variable with mean 0 and variance $\sigma^{2}, U$ is a non-negative random variable with density $(2.1), Z_{0}$ and $\mathrm{U}$ are independent each other, and

$$
\lambda=\left(E U^{2}\right)^{-1 / 2}=\left(\ln \alpha_{1}-\ln \alpha_{2}\right)^{1 / 2}\left(\alpha_{2}^{-1}-\alpha_{1}^{-1}\right)^{-1 / 2} .
$$

For a K-differenced random variable $Z$, we have its mean as

$$
E Z=\lambda E U E Z_{0}+\mu=\mu,
$$

and its variance as

$$
\operatorname{Var}(Z)=\operatorname{Var}\left(\lambda U Z_{0}\right)=\lambda^{2} E U^{2} E Z_{0}^{2}=\sigma^{2} .
$$




\subsection{Density function}

In this section we are going to derive the density function of the K-differenced random variable $Z$ defined by $(2.2)$.

The cumulative distribution function of $Z$ is

$$
\begin{aligned}
& P(Z \leq z) \\
& =\mathrm{P}\left(\lambda U Z_{0}+\mu \leq z\right) \\
& =\int_{0}^{\infty} P\left(\lambda u Z_{0}+\mu \leq z\right) f_{U}(u) \quad d u \\
& =\int_{0}^{\infty} P\left(Z_{0} \leq \frac{z-\mu}{\lambda u}\right) f_{U}(u) \quad d u, \quad z \in \mathbb{R} .
\end{aligned}
$$

Taking the derivative, we obtain the density function of $Z$ as follows,

$$
\begin{aligned}
& f_{Z}(z)=\frac{d}{d z} P(Z \leq z) \\
& =\frac{1}{(2 \pi)^{1 / 2} \sigma} \int_{0}^{\infty} u^{-1} \exp \left(-\frac{(z-\mu)^{2}}{2 \lambda^{2} \sigma^{2} u^{2}}\right) f_{U}(u) d u \\
& =(2 \pi)^{-1 / 2} \lambda^{-1} \sigma^{-1 / 2} \int_{0}^{\infty} u^{-1} \exp \left(-\frac{(z-\mu)^{2}}{2 \lambda^{2} u^{2} \sigma^{2}}\right) f_{U}(u) d u \\
& =\frac{2(2 \pi)^{-1 / 2} \lambda^{-1} \sigma^{-1 / 2}}{\ln \alpha_{1}-\ln \alpha_{2}} \int_{0}^{\infty} u^{-2} \exp \left(-\frac{(z-\mu)^{2}}{2 \lambda^{2} \sigma^{2} u^{2}}\right)\left\{\exp \left(-\alpha_{2} u^{2}\right)-\exp \left(-\alpha_{1} u^{2}\right)\right\} d u \\
& =\frac{(2 \pi)^{-1 / 2} \lambda^{-1} \sigma^{-1 / 2}}{\ln \alpha_{1}-\ln \alpha_{2}} \int_{0}^{\infty} v^{-3 / 2} \exp \left(-\frac{(z-\mu)^{2}}{2 \lambda^{2} \sigma^{2} v}\right)\left\{\exp \left(-\alpha_{2} v\right)-\exp \left(-\alpha_{1} v\right)\right\} d v
\end{aligned}
$$




$$
=\frac{1}{\left(\ln \alpha_{1}-\ln \alpha_{2}\right)|z-\mu|}\left\{\exp \left(-\frac{\left(2 \alpha_{2}\right)^{1 / 2}|z-\mu|}{\sigma \lambda}\right)-\exp \left(-\frac{\left(2 \alpha_{1}\right)^{1 / 2}|z-\mu|}{\sigma \lambda}\right)\right\}, \quad z \in \mathbb{R},
$$

where the fourth equality is obtained by making a transform $u=v^{1 / 2}$ and the last equality is obtained by using the formula

$$
\int_{0}^{\infty} t^{-3 / 2} \exp \left(-\frac{\alpha}{4 t}\right) \exp (-\beta t) d t=2 \pi^{1 / 2} \alpha^{-1 / 2} e^{-\alpha^{1 / 2} \beta^{-1 / 2}}, \quad \beta>0
$$

(see Bateman (1954) formula 28, p. 146).

The density function of $Z$ is illustrated in Figures 2.2-2.5 with various selection of the parameters $\alpha_{1}, \alpha_{2}, \sigma$, and $\mu$.

Figure 2.4 illustrates the effect of the parameter $\alpha_{1}$, with $\alpha_{2}$ fixed. When $\alpha_{1}$ gets larger, the peak of the density function gets lower, and the tails gets wider.

Figure 2.5 illustrates the effect of the parameter $\alpha_{2}$, with $\alpha_{1}$ fixed. As $\alpha_{2}$ gets larger, the peak of the density function gets higher, and the tails gets narrow.

The case where $\mu=0$ and $\sigma=1$ is called the standard K-differenced distribution. The standard K-differenced density function is

$$
\begin{aligned}
f(z)=\frac{1}{\left(\ln \alpha_{1}-\ln \alpha_{2}\right)|z|}\left\{\exp \left(-\left(\frac{\alpha_{2}^{-1}-\alpha_{1}^{-1}}{\ln \alpha_{1}-\ln \alpha_{2}}\right)^{1 / 2}\left(2 \alpha_{2}\right)^{1 / 2}|z|\right)\right. \\
\\
\left.\quad-\exp \left(-\left(\frac{\alpha_{2}^{-1}-\alpha_{1}^{-1}}{\ln \alpha_{1}-\ln \alpha_{2}}\right)^{1 / 2}\left(2 \alpha_{1}\right)^{1 / 2}|z|\right)\right\},
\end{aligned}
$$




$$
-\infty<z<\infty
$$

and the cumulative distribution function is

$$
\begin{aligned}
\frac{1}{\left(\ln \alpha_{1}-\ln \alpha_{2}\right)} \int_{-\infty}^{z} \frac{1}{|t|}\{\exp ( & \left.-\left(\frac{\alpha_{2}^{-1}-\alpha_{1}^{-1}}{\ln \alpha_{1}-\ln \alpha_{2}}\right)^{1 / 2}\left(2 \alpha_{2}\right)^{1 / 2}|t|\right) \\
& \left.-\exp \left(-\left(\frac{\alpha_{2}^{-1}-\alpha_{1}^{-1}}{\ln \alpha_{1}-\ln \alpha_{2}}\right)^{1 / 2}\left(2 \alpha_{1}\right)^{1 / 2}|t|\right)\right\} d t, \quad z \in \mathbb{R} .
\end{aligned}
$$

Unfortunately, the last integral does not have a simple closed form. It can be evaluated numerically.

\subsection{Characteristic function}

In this section we derive the characteristic function of K-differenced random variable (2.2).

$$
\begin{aligned}
& E \exp \{i Z \omega\} \\
& =\mathrm{E}\left\{\exp \left(i\left(\lambda U Z_{0}+\mu\right) \omega\right)\right\} \\
& =\int_{0}^{\infty} E\left\{\exp \left(i\left(\lambda U Z_{0}+\mu\right) \omega\right)\right\} f_{U}(u) d u \\
& =\frac{2 \exp (i \mu \omega)}{\ln \alpha_{1}-\ln \alpha_{2}} \int_{0}^{\infty} u^{-1} \exp \left(-\frac{\lambda^{2} u^{2}}{2} \sigma^{2} \omega^{2}\right)\left\{\exp \left(-\alpha_{2} u^{2}\right)-\exp \left(\alpha_{1} u^{2}\right)\right\} d u \\
& =\frac{\exp (i \mu \omega)}{\ln \alpha_{1}-\ln \alpha_{2}} \int_{0}^{\infty} v^{-1} \exp \left(-\frac{\lambda^{2} v}{2} \sigma^{2} \omega^{2}\right)\left\{\exp \left(-\alpha_{2} v\right)-\exp \left(\alpha_{1} v\right)\right\} d v \\
& =\frac{\exp (i \mu \omega)}{\ln \alpha_{1}-\ln \alpha_{2}}\left[\ln \left(\frac{\lambda^{2}}{2} \sigma^{2} \omega^{2}+\alpha_{1}\right)-\ln \left(\frac{\lambda^{2}}{2} \sigma^{2} \omega^{2}+\alpha_{2}\right)\right], \omega \in \mathbb{R},
\end{aligned}
$$


where the fourth equality is obtained by letting $u^{2}=v$, and the last one by using formula (see Bateman (1954), formula 4, p.144),

$$
\int_{0}^{\infty} \omega^{-1} \exp (-x \omega)\left(\exp \left(-\alpha_{2} \omega\right)-\exp \left(-\alpha_{1} \omega\right)\right) d \omega=\ln \left(x+\alpha_{1}\right)-\ln \left(x+\alpha_{2}\right) .
$$

In the special case where $\alpha_{1}$ tends to $\alpha_{2}$, we obtain the limit characteristic function as, by using L'Hpital's rule

$$
\begin{aligned}
& \lim _{\alpha_{1} \rightarrow \alpha_{2-}-\exp (i \mu \omega)}\left[\ln \alpha_{1}-\ln \alpha_{2}\left(\frac{\lambda^{2}}{2} \sigma^{2} \omega^{2}+\alpha_{1}\right)-\ln \left(\frac{\lambda^{2}}{2} \sigma^{2} \omega^{2}+\alpha_{2}\right)\right] \\
& =\lim _{\alpha_{1} \rightarrow \alpha_{2-}} \exp (i \mu \omega)\left\{\frac{\frac{\lambda^{2} \sigma^{2} \omega^{2}}{2}+\alpha_{1}}{\frac{1}{\alpha_{1}}}\right\} \\
& =\frac{\exp (i \mu \omega)}{1+\frac{\lambda^{2} \sigma^{2}}{2 \alpha_{2}} \omega^{2}}, \quad \omega \in \mathbb{R},
\end{aligned}
$$

which is the characteristic function of the double exponential or Laplace distribution.

\subsection{Simulation}

In this section we review some known simulation methods first, and then apply them to simulate random numbers from the K-differenced distribution.

Monte Carlo Approxmation The Monte Carol method supplies approximations to solution of a variety of mathematical problems by performing statistical sampling experimentation on computer. In this dissertation we will use the MatLab programing and packages to derive our approximations and to test the simulation performance. 
Generating a random sample is a fundamental advantage of every Monte Carlo experimentation. If we have an expression that needs to be estimated, then Monte Carlo procedure generates random numbers from an uniform distribution and this would be sufficient. There are many Monte Carlo methods that can be used for approximation or generating data. The method that works for our situation is the acceptance-rejection method, since the density function $f(x)$ can be calculated.

Acceptance-Rejection Method Acceptance-rejection method is a Monte Carlo method to draw independent samples from a target random variable $X$ with probability density $f(x)$, where $x \in X \subset R^{k}$. If $f(x)$ can be determined, then we can use the acceptancerejection sampling to obtain random numbers drawn from exactly the target distribution. This method depends on some sampling generated from an easier distribution called a proposal distribution, and on validating the sampling probability through random rejection of some that sampling generated.

Suppose that $X$ and $Y$ are random variables with density $f$ and $g$ respectively, and there exists a constant $c$ such that

$$
\frac{f(t)}{g(t)} \leq c
$$

for all $t$ such that $g(t)>0$. The acceptance-rejection method or (rejection method) can be applied to generate the random variable number as follows.

\section{Algorithm of Sampling Rejection}

1. Choose a density $g(x)$.

2. Find a constant $\mathrm{c}$ such that $f(x) / g(x) \leq c$ for all $x$.

3. Generate a random number $y$ from the density $g$. 
4. Generate a $\operatorname{Unif}(0,1)$ random number u.

5. If $u \leq \frac{f(y)}{c g(y)}$ then accept $y$ and take $x=y$; otherwise reject and go back to Step 3 .

Effectiveness Measurement For the simulated data, the histogram will be implemented, and be compared with plot of the density function of the K-differenced random variable. Both figures need to have same shape.

Generating K-differenced random numbers Let $f(x)$ be the density function (2.3) of a standard K-differenced distribution with $\alpha_{1}=3.7$ and $\alpha_{2}=12.19$. We apply the acceptancerejection method to generate random numbers from this distribution.

Choose $g(x)$ to be a Laplace density function

$$
g(x)=\frac{1}{2} e^{-|x|}, \quad x \in \mathbb{R} .
$$

We can find $c=\max \left(\frac{f(x)}{g(x)}\right)$, so that

$$
\frac{f(x)}{g(x)} \leq c, \quad x \in \mathbb{R} .
$$

This method is illustrated in Figure 2.6. A random number $y$ from $g(y)$ is accepted if

$$
\frac{f(y)}{c g(y)}>u,
$$

where $u$ is a random number generated from a uniform $(0,1)$ distribution. 
The procedure is performed using MaLab. The MatLab codes are given in Appendex A. Here a zero-mean Laplace distribution is used as the proposal distribution. This distribution is scaled by a factor $c=1.4771$, determined from $f(x)$ and $g(x)$ to ensure that the proposal distribution covers $f(x)$. In Figure 2.4 we accept the sample with the probability associated with green line segment and reject the sample associated with red line segment.

After generating the data we plot them via a histogram plot in Figure 2.7 and compare it with density function in Figure 2.2. It looks like a good approximation. Figure 2.8 is a box plot of the simulated data.

The acceptance-rejection method can be used for simulation of data with other choices of $\alpha_{1}$ and $\alpha_{2}$. Figure 2.9 is the histogram of the simulated data with $\alpha_{1}=6$ and $\alpha_{2}=22$.

\subsection{Maximum likelihood estimation}

Suppose that a set of observations $z_{1}, \ldots, z_{n}$ is taken from a population with a Kdifferenced distribution having parameters $\mu, \sigma, \alpha_{1}, \alpha_{2}$. we want to estimate these parameters by the maximum likelihood method.

The log-likelihood function is

$$
\begin{aligned}
& \ell\left(\mu, \sigma, \alpha_{1}, \alpha_{2}\right)=\sum_{i=1}^{n} \log f\left(z_{i} ; \mu, \sigma, \alpha_{1}, \alpha_{2}\right) \\
& =\sum_{i=1}^{n} \log \left\{\frac{1}{\left(\ln \alpha_{1}-\ln \alpha_{2}\right)\left|z_{i}-\mu\right|}\left(\exp \left(-\frac{\left(2 \alpha_{2}\right)^{1 / 2}\left|z_{i}-\mu\right|}{\sigma \lambda}\right)-\exp \left(-\frac{\left(2 \alpha_{1}\right)^{1 / 2}\left|z_{i}-\mu\right|}{\sigma \lambda}\right)\right)\right\}
\end{aligned}
$$

where $\lambda=\left(\log \alpha_{1}-\log \alpha_{2}\right)^{1 / 2}\left(\alpha_{2}^{-1}-\alpha_{1}^{-1}\right)^{-1 / 2}$. Since it is hard to maximize this function manually, we have to maximize it numerically (using optim in MatLab) to obtain the maxi- 
mum likelihood estimators.

Nelder-Mead Method This method was proposed by Nelder and Mead (1965). It is a simplex maximization method for optimization. Also, it can be considered as a technique for minimizing an objective function in a multi-dimensional space, and does not ask for derivatives. This method is simple and stable, for example, this method can be obtained by evaluating the handled function of maximum likelihood with the given initial estimate, by using the "fminsearch" MathLab command, for the simplex search. Then this step is repeated until the desired value obtained.

For the simulation data that are generated from the K-differenced distribution in Section 2.4 we can obtain the maximum likelihood estimators of the parameters by using the Nelder-Mead method for the optimization. Tables 2.1 and 2.2 show the true values and the estimators of the parameters.

As we can see from Table 2.1 and Table 2.2, the maximum likelihood estimation procedure works pretty well, and Figures 2.7 and 2.9 also indicate a good fit. 


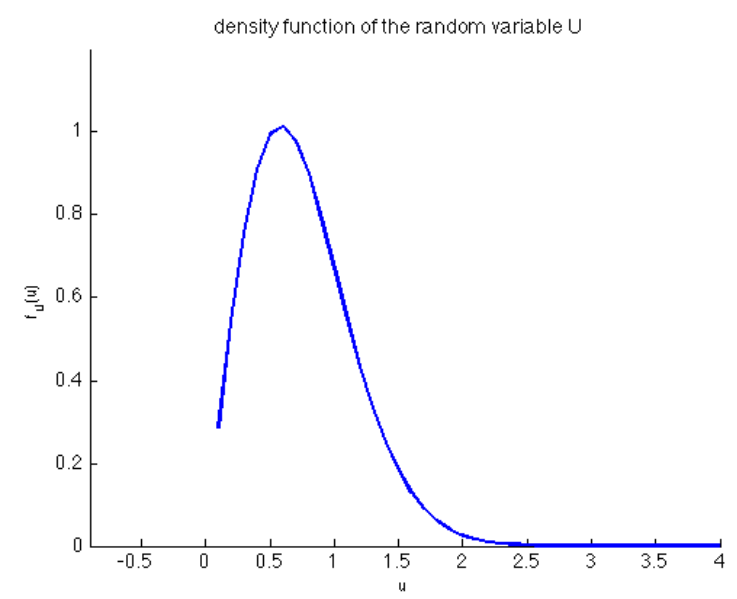

Figure 2.1: The density function $f_{U}(u)$ with $\alpha_{1}=1$ and $\alpha_{2}=2$

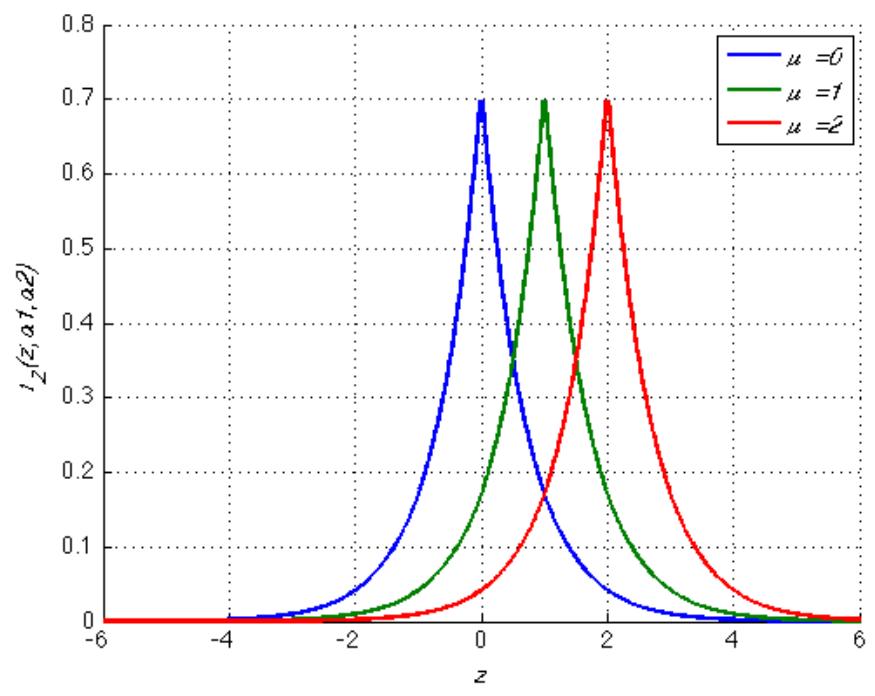

Figure 2.2: K-differenced density function with $\mu=0,1,2, \sigma=1, \alpha_{1}=5$ and $\alpha_{2}=6$.

Table 2.1: K-differenced (using MLE) fitted to the simulated data with $\alpha_{1}=3.7$ and $\alpha_{2}=$ 12.19 .

\begin{tabular}{|l|l|l|l|l|}
\hline Parameters & $\mu$ & $\sigma$ & $\alpha_{1}$ & $\alpha_{2}$ \\
\hline True value & 0 & 1 & 3.7 & 12.19 \\
\hline MLE & 0.0001 & 1.0418 & 3.5886 & 12.7226 \\
\hline
\end{tabular}




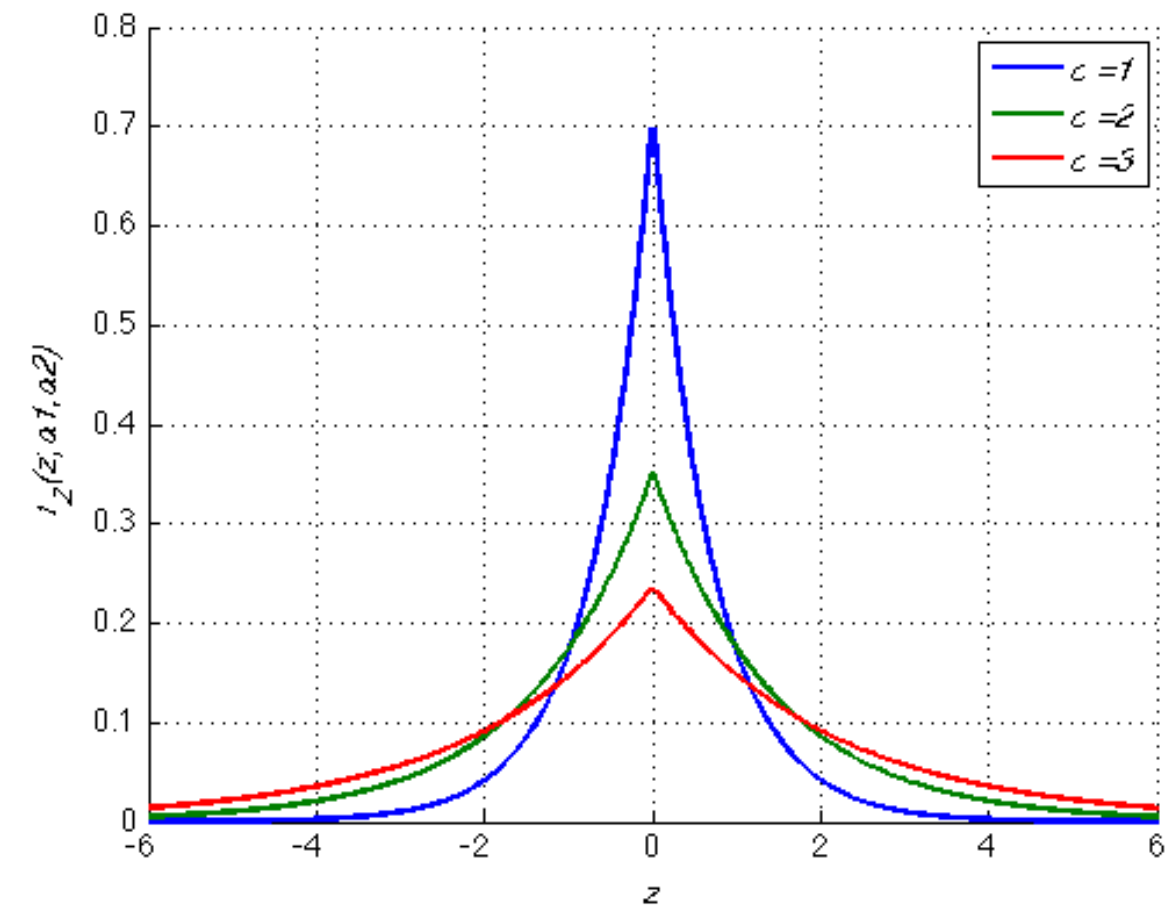

Figure 2.3: K-differenced density function with $\mu=0, \sigma=1,2,3$, and $\alpha_{1}=5$ and $\alpha_{2}=6$

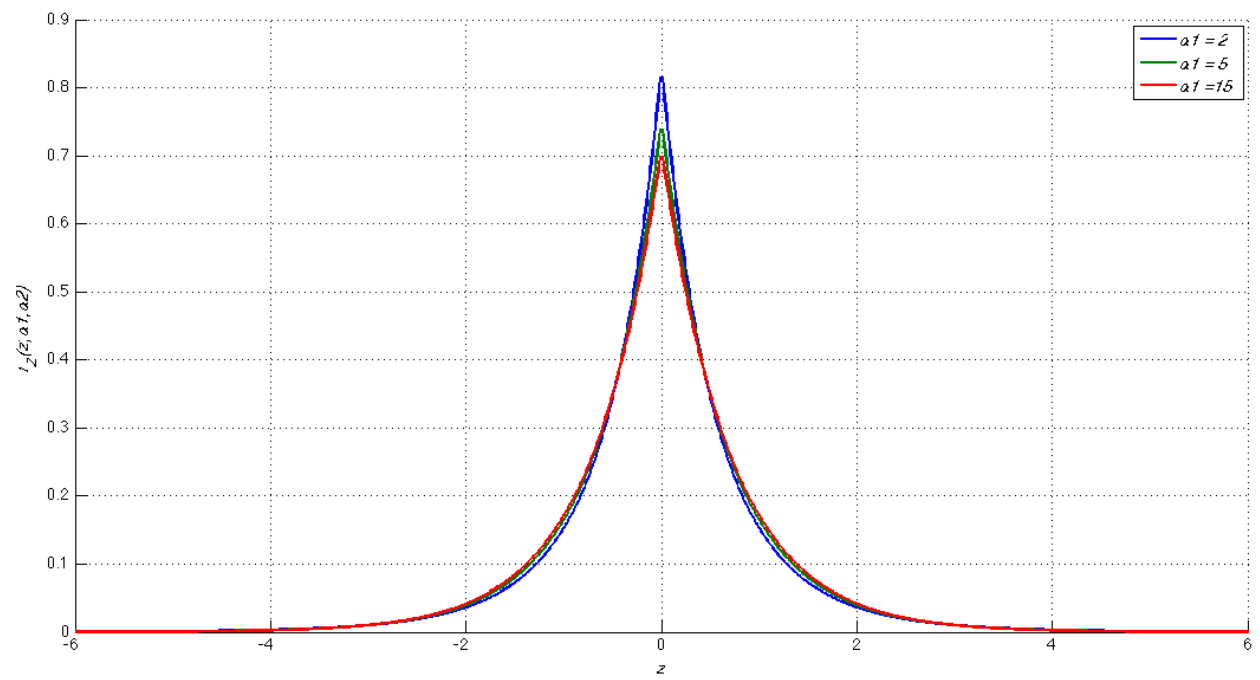

Figure 2.4: K-differenced density function with $\mu=0, \sigma=1, \alpha_{2}=20$ and $\alpha_{1}=2,5,15$ 


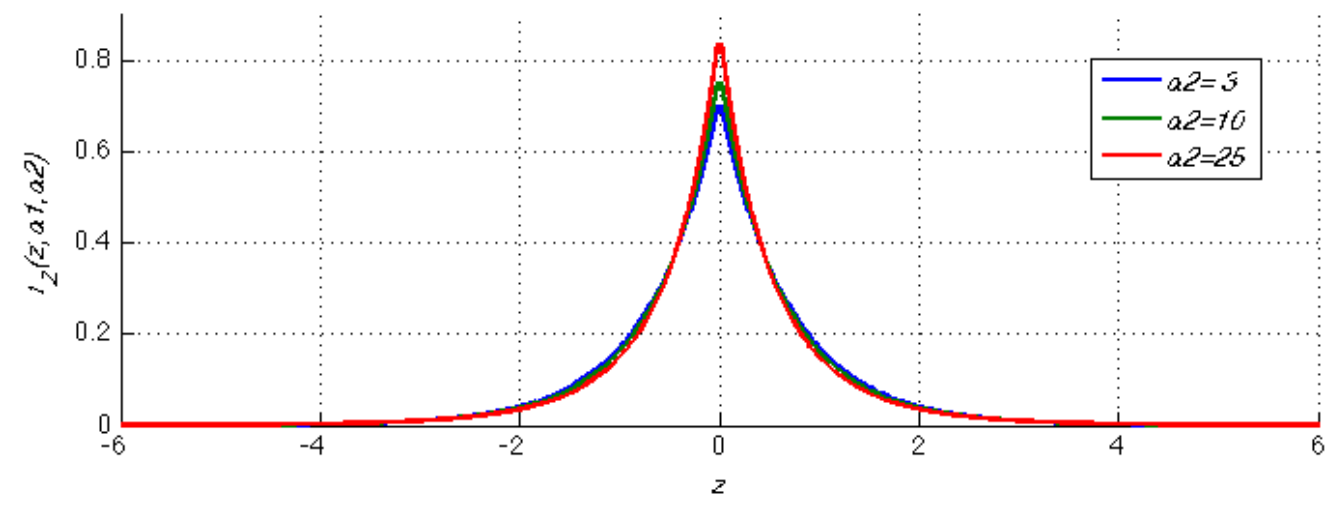

Figure 2.5: K-differenced density curves with $\mu=0, \sigma=1, \alpha 1=2$ and $\alpha 2=3,10,25$

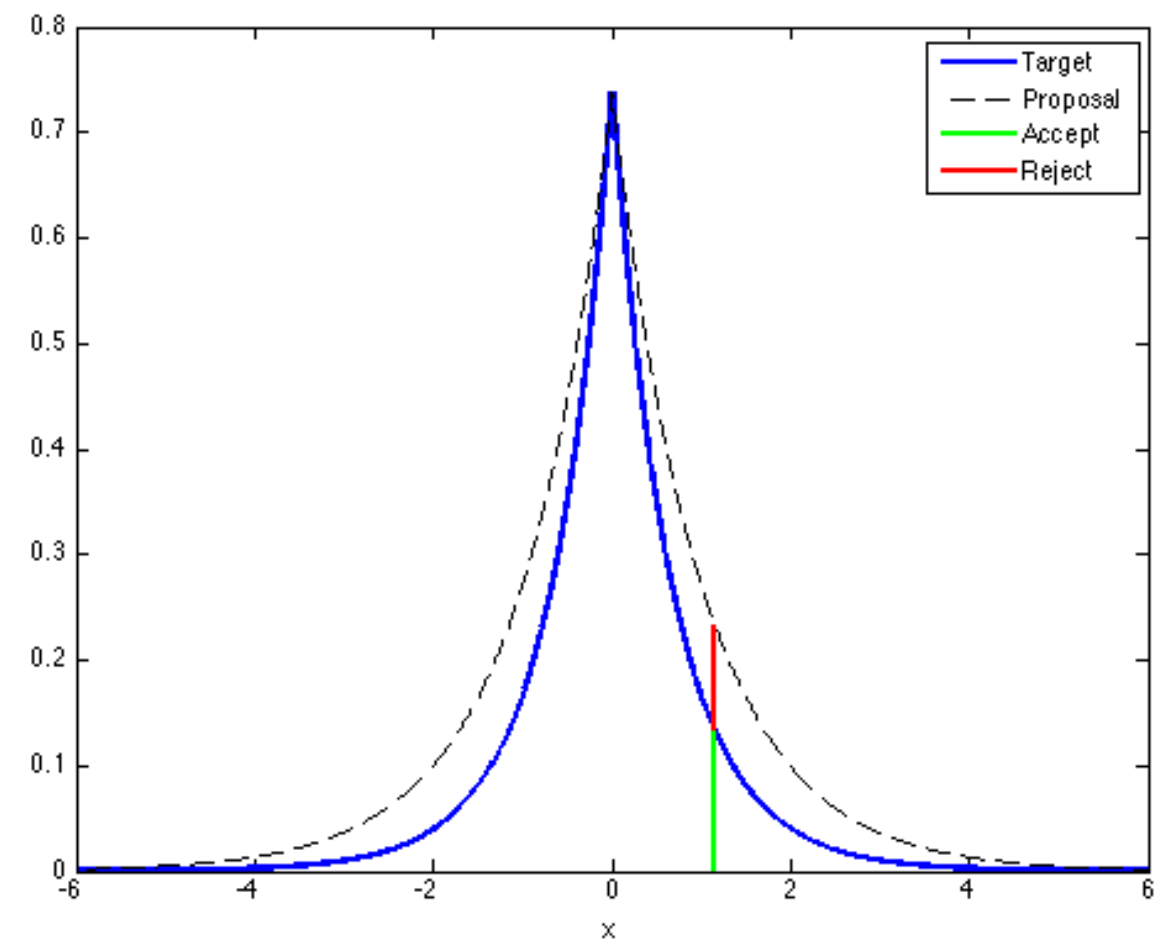

Figure 2.6: Rejection sampling with a Laplace proposal distribution.

Table 2.2: K-differenced (using MLE) fitted to the simulated data with $\alpha_{1}=6$ and $\alpha_{2}=22$.

\begin{tabular}{|l|l|l|l|l|}
\hline Parameters & $\mu$ & $\sigma$ & $\alpha_{1}$ & $\alpha_{2}$ \\
\hline True value & 0 & 1 & 6 & 22 \\
\hline MLE & 0.0195 & 0.9787 & 6.0391 & 22.6229 \\
\hline
\end{tabular}




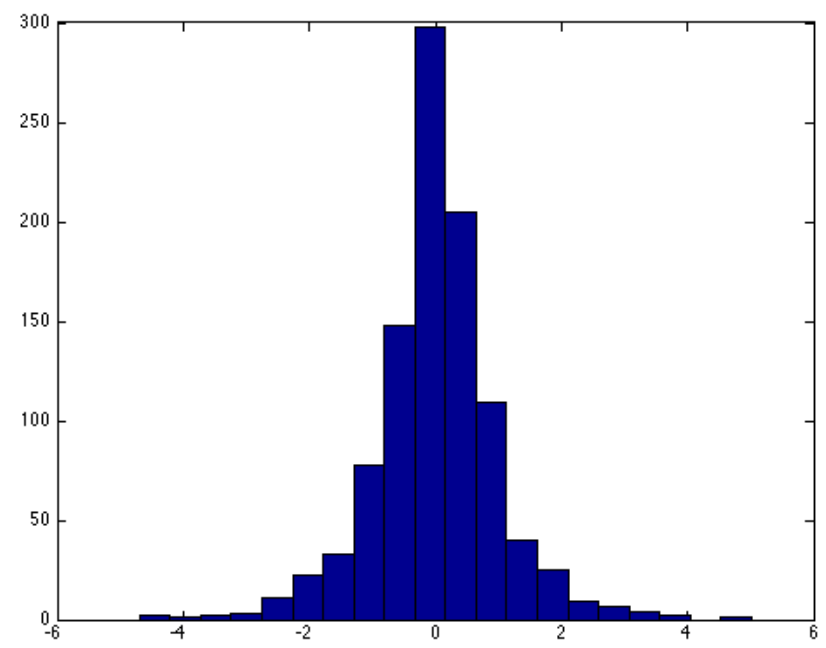

Figure 2.7: Histogram of the simulated K-differenced with $\alpha_{1}=3.7$ and $\alpha_{2}=12.19$ data

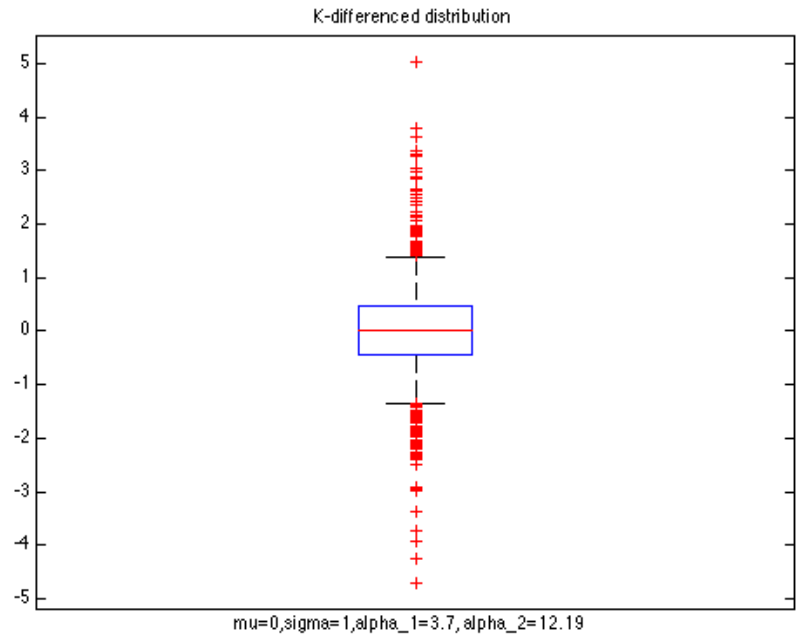

Figure 2.8: Box plot of the simulated K-differenced data with $\alpha_{1}=3.7$ and $\alpha_{2}=12.19$. 


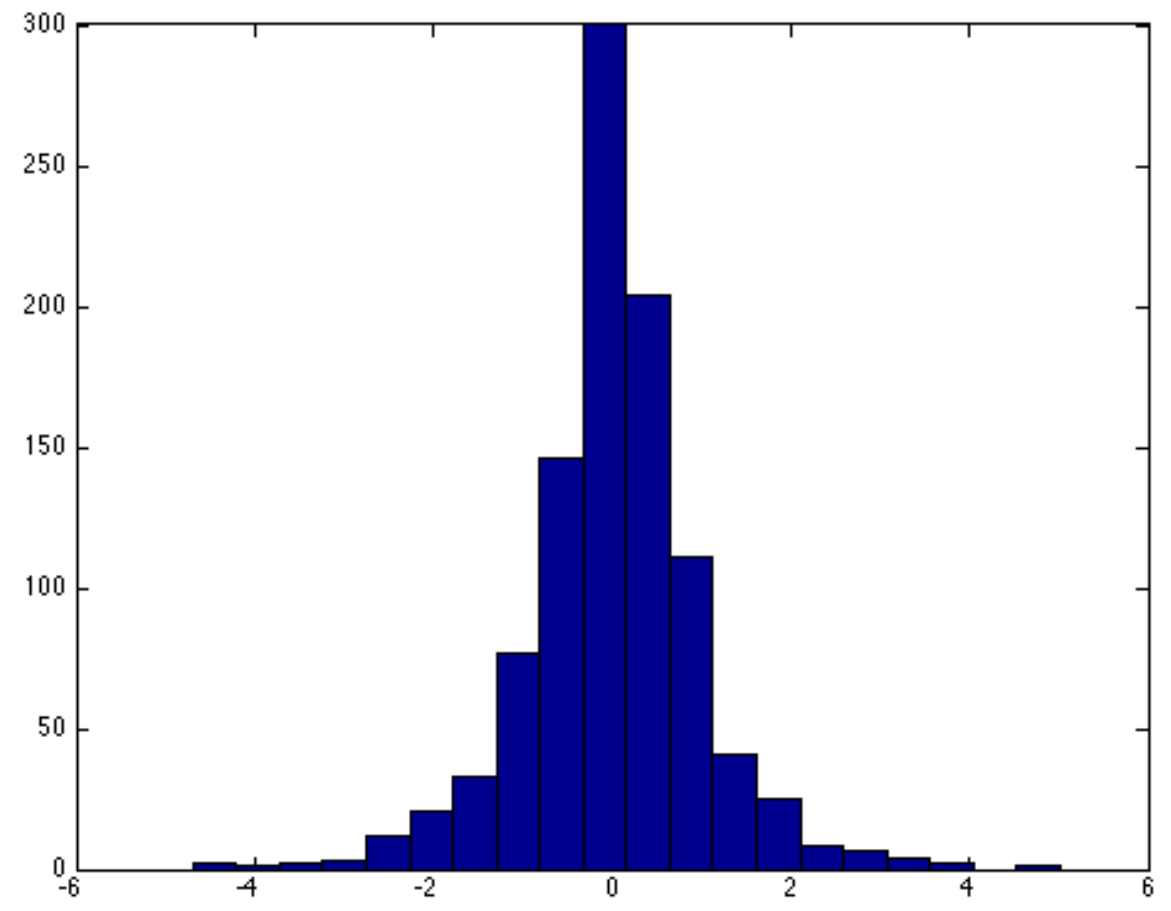

Figure 2.9: Histogram of the simulated K-differenced $\left(\alpha_{1}=6\right.$ and $\left.\alpha_{2}=22\right)$ data. 


\section{CHAPTER 3}

\section{K-differenced Random Vector}

This chapter defines a K-differenced random vector through a stochastic representation in Section 3.1, and derives its density function and characteristic function in Section 3.2 and 3.3, respectively. Simulations are made using several methods in Section 3.4. The maximum likelihood estimators of the parameters are found numerically for the simulated data in Section 3.5 with MatLab codes in Appendix B.

\subsection{Stochastic representation}

This section provides a construction of the K-differenced random vector via a nonnegative random variable with density (2.1) and a normal random vector, which are assumed to be independent.

We define an n-variate K-differenced random vector by

$$
\mathbf{Z}=\lambda U \mathbf{Z}_{0}+\boldsymbol{\mu},
$$

where $\boldsymbol{\mu}$ is a parameter vector, $\lambda=\left(E U^{2}\right)^{-1 / 2}$, random variable $U$ has density $(2.1), \mathbf{Z}_{0}$ is an n-variate normal random vector with mean $\mathbf{0}$ and variance-covariance matrix $\boldsymbol{\Sigma}$, and $U$ and $\mathbf{Z}_{\mathbf{0}}$ are independent.

For a K-differenced random vector $\mathbf{Z}$, we have its mean vector as 


$$
E \mathbf{Z}=\lambda E U E \mathbf{Z}_{0}+\boldsymbol{\mu}=\boldsymbol{\mu}
$$

and its variance-covariance matrix as

$$
\operatorname{cov}(\mathbf{Z}, \mathbf{Z})=\lambda^{2} \operatorname{cov}\left(U \mathbf{Z}_{0}, U \mathbf{Z}_{0}\right)=\lambda^{2} E U^{2} \boldsymbol{\Sigma}=\boldsymbol{\Sigma} .
$$

\subsection{Density function}

There is no closed form for the cumulate distribution function of the K-differenced random vector $\mathbf{Z}$ formed by the stochastic representation (3.1). Suppose that $\boldsymbol{\Sigma}$ is strictly positive definite. We find the density function of $\mathbf{Z}=\left(Z_{1}, \ldots, Z_{n}\right)^{\prime}$ as $f\left(\mathbf{z} ; \boldsymbol{\mu}, \boldsymbol{\Sigma}, \alpha_{1}, \alpha_{2}\right)=$

$$
\begin{aligned}
& \frac{\partial^{n}}{\partial z_{1} \cdots \partial z_{n}} \mathrm{P}\left(\mathrm{Z}_{1} \leq z_{1}, \cdots, Z_{n} \leq z_{n}\right) \\
& =\frac{\partial^{n}}{\partial z_{1} \cdots \partial z_{n}} \mathrm{P}\left(\lambda U Z_{01}+\mu_{1} \leq z_{1}, \cdots, \lambda U Z_{0 n}+\mu_{n} \leq z_{n}\right) \\
& =\frac{\partial^{n}}{\partial z_{1} \cdots \partial z_{n}} \int_{0}^{\infty} P\left(\lambda u Z_{01}+\mu_{1} \leq z_{1}, \cdots, \lambda u Z_{0 n}+\mu_{n} \leq z_{n}\right) f_{U}(u) d u \\
& =\int_{0}^{\infty} \frac{\partial^{n}}{\partial z_{1} \cdots \partial z_{n}} \mathrm{P}\left(\mathrm{Z}_{01} \leq \frac{z_{1}-\mu_{1}}{\lambda u}, \cdots, Z_{0 n} \leq \frac{z_{n}-\mu_{n}}{\lambda u}\right) f_{U}(u) d u \\
& =(2 \pi)^{-n / 2} \lambda^{-n}|\boldsymbol{\Sigma}|^{-1 / 2} \int_{0}^{\infty} u^{-n} \exp \left(-\frac{1}{2 \lambda^{2} u^{2}}(\mathbf{z}-\boldsymbol{\mu})^{\prime} \boldsymbol{\Sigma}^{-1}(\mathbf{z}-\boldsymbol{\mu})\right) f_{U}(u) d u, \\
& =\frac{2}{\left(\ln \alpha_{1}-\ln \alpha_{2}\right)}(2 \pi)^{-n / 2} \lambda^{-n}|\boldsymbol{\Sigma}|^{-1 / 2} \int_{0}^{\infty} u^{-n-1} \exp \left(-\frac{1}{2 \lambda^{2} u^{2}}\left((\mathbf{z}-\boldsymbol{\mu})^{\prime} \boldsymbol{\Sigma}^{-1}(\mathbf{z}-\boldsymbol{\mu})\right)\right) \\
& \times\left\{\exp \left(-\alpha_{2} u^{2}\right)-\exp \left(-\alpha_{1} u^{2}\right)\right\} d u \\
& =\frac{1}{\left(\ln \alpha_{1}-\ln \alpha_{2}\right)}(2 \pi)^{-n / 2} \lambda^{-n}|\boldsymbol{\Sigma}|^{-1 / 2} \int_{0}^{\infty} v^{-n / 2-1} \exp \left(-\frac{1}{2 \lambda^{2} v}\left((\mathbf{z}-\boldsymbol{\mu})^{\prime} \boldsymbol{\Sigma}^{-1}(\mathbf{z}-\boldsymbol{\mu})\right)\right) \\
& \times\left\{\exp \left(-\alpha_{2} v\right)-\exp \left(-\alpha_{1} v\right\} d v\right.
\end{aligned}
$$




$$
\begin{aligned}
=\mathrm{c}_{0}|\boldsymbol{\Sigma}|^{-1 / 2}\left\{\left(\frac{\alpha_{2}}{(\mathbf{z}-\boldsymbol{\mu})^{\prime} \mathbf{\Sigma}^{-1}(\mathbf{z}-\boldsymbol{\mu})}\right)^{n / 4} K_{\frac{-n}{2}}\left(\lambda^{-1}\left(2 \alpha_{2}\right)^{1 / 2}\left((\mathbf{z}-\boldsymbol{\mu})^{\prime} \boldsymbol{\Sigma}^{-1}(\mathbf{z}-\boldsymbol{\mu})\right)^{1 / 2}\right)\right. \\
\left.\quad-\left(\frac{\alpha_{1}}{(\mathbf{z}-\boldsymbol{\mu})^{\prime} \mathbf{\Sigma}^{-1}(\mathbf{z}-\boldsymbol{\mu})}\right)^{n / 4} K_{\frac{-n}{2}}\left(\lambda^{-1}\left(2 \alpha_{1}\right)^{1 / 2}\left((\mathbf{z}-\boldsymbol{\mu})^{\prime} \boldsymbol{\Sigma}^{-1}(\mathbf{z}-\boldsymbol{\mu})\right)^{1 / 2}\right)\right\}
\end{aligned}
$$

where last equality is obtained by making the substitution $v=u^{2}$ and the formula

(see Bateman (1954), formula 29, p.146),

$$
\int_{0}^{\infty} \nu^{d-1} \exp \left(\frac{-a \nu^{-1}}{4}-t \nu\right) \mathrm{d} \nu=2\left(\frac{a}{4 t}\right)^{d / 2} K_{d}\left(a^{-1 / 2} t^{-1 / 2}\right)
$$

is used, where $K_{\tau}($.$) is a modified Bessel function of the second type of order \tau$ and

$$
c_{0}=\frac{2^{1-n / 4} \pi^{-n / 2} \lambda^{-n / 2}}{\ln \alpha_{1}-\ln \alpha_{2}}
$$

From the property of Bessel functions that $K_{\tau}(x)=K_{-\tau}(x)$, for any $\tau>0$ (see Kotz et al. (2001), page 317), we see that the density function $f\left(\mathbf{z} ; \boldsymbol{\mu}, \boldsymbol{\Sigma}, \alpha_{1}, \alpha_{2}\right)$ of the K-differenced random vector $\mathbf{Z}$ is

$$
\begin{gathered}
f\left(\mathbf{z} ; \boldsymbol{\mu}, \boldsymbol{\Sigma}, \alpha_{1}, \alpha_{2}\right)=c_{0}|\boldsymbol{\Sigma}|^{-1 / 2}\left\{\left(\frac{\alpha_{2}}{(\mathbf{z}-\boldsymbol{\mu})^{\prime} \mathbf{\Sigma}^{-1}(\mathbf{z}-\boldsymbol{\mu})}\right)^{n / 4} K_{\frac{n}{2}}\left(\lambda^{-1}\left(2 \alpha_{2}\right)^{1 / 2}\left((\mathbf{z}-\boldsymbol{\mu})^{\prime} \boldsymbol{\Sigma}^{-1}(\mathbf{z}-\boldsymbol{\mu})\right)^{1 / 2}\right)\right. \\
\left.-\left(\frac{\alpha_{1}}{(\mathbf{z}-\boldsymbol{\mu})^{\prime} \mathbf{\Sigma}^{-1}(\mathbf{z}-\boldsymbol{\mu})}\right)^{n / 4} K_{\frac{n}{2}}\left(\lambda^{-1}\left(2 \alpha_{1}\right)^{1 / 2}\left((\mathbf{z}-\boldsymbol{\mu})^{\prime} \boldsymbol{\Sigma}^{-1}(\mathbf{z}-\boldsymbol{\mu})\right)^{1 / 2}\right)\right\} \\
\mathbf{z} \in \mathbb{R}^{n} .
\end{gathered}
$$


Because the density function (3.2) is the difference of two Bessel functions, or K functions, we call it the K-differenced density function, and the random vector as a K-differenced random vector.

Next, we consider the bivariate case with $n=2$ in (3.2), where $\boldsymbol{\Sigma}$ is a strictly positive definite matrix,

$$
\boldsymbol{\Sigma}=\left(\begin{array}{cc}
\sigma_{1}^{2} & \sigma_{1} \sigma_{2} \rho \\
\sigma_{1} \sigma_{2} \rho & \sigma_{2}^{2}
\end{array}\right)
$$

with $\sigma_{1}>0, \sigma_{2}>0$, and $|\rho|<1$. In this case, the bivariate K-differenced density $f\left(z_{1}, z_{2}\right)$ takes the form

$$
\begin{gathered}
\frac{1}{\pi \lambda\left(\ln \alpha_{1}-\ln \alpha_{2}\right) \sigma_{1} \sigma_{2} \sqrt{1-\rho^{2}}}\left\{\left(\frac{2 \alpha_{2}\left(1-\rho^{2}\right)}{z_{1}^{2} / \sigma_{1}^{2}-2 \rho z_{1} z_{2} /\left(\sigma_{1} \sigma_{2}\right)+z_{2}^{2} / \sigma_{2}^{2}}\right)^{1 / 2} K_{1}\left(\lambda^{-1}\left(2 \alpha_{2}\right)^{1 / 2}\left(\frac{\frac{z_{1}^{2}}{\sigma_{1}^{2}}-\frac{2 \rho z_{1} z_{2}}{\left(\sigma_{1} \sigma_{2}\right.}+\frac{z_{2}^{2}}{\sigma_{2}^{2}}}{1-\rho^{2}}\right)^{1 / 2}\right)\right. \\
\left.-\left(\frac{2 \alpha_{1}\left(1-\rho^{2}\right)}{\left(z_{1}^{2} / \sigma_{1}^{2}-2 \rho z_{1} z_{2} /\left(\sigma_{1} \sigma_{2}\right)+z_{2}^{2} / \sigma_{2}^{2}\right)}\right)^{1 / 2} K_{1}\left(\lambda^{-1}\left(2 \alpha_{1}\right)^{1 / 2}\left(\frac{z_{1}^{2}}{\sigma_{1}^{2}-\frac{2 \rho z_{1} z_{2}}{\left(\sigma_{1} \sigma_{2}\right)}} \frac{z_{2}^{2}}{1-\rho^{2}}\right)^{2}\right)\right\}, \\
-\infty<z_{1}, z_{2}<\infty .
\end{gathered}
$$

In particular, the standard bivariate K-differenced density function, with $\boldsymbol{\Sigma}=\mathbf{I}_{2}$, is given by

$$
\begin{aligned}
f\left(z_{1}, z_{2}\right)=\frac{1}{\pi \lambda\left(\ln \alpha_{1}-\ln \alpha_{2}\right)} & \left\{\left(\frac{2 \alpha_{2}}{z_{1}^{2}+z_{2}^{2}}\right)^{1 / 2} K_{1}\left(\lambda^{-1}\left(2 \alpha_{2}\right)^{1 / 2}\left(z_{1}^{2}+z_{2}^{2}\right)^{1 / 2}\right)\right. \\
& \left.-\left(\frac{2 \alpha_{1}}{z_{1}^{2}+z_{2}^{2}}\right)^{1 / 2} K_{1}\left(\lambda^{-1}\left(2 \alpha_{1}\right)^{1 / 2}\left(z_{1}^{2}+z_{2}^{2}\right)^{1 / 2}\right)\right\},\left(z_{1}, z_{2}\right)^{\prime} \in \mathbb{R}^{2} .
\end{aligned}
$$

Figure 3.1 is a plot of the standard bivariate K-differenced density function. 


\subsection{Characteristic function}

In this section we are going to find the characteristic function of the K-differenced random vector $\mathbf{Z}$ defined by (3.1).

Notice that the characteristic function of the normal random vector $\mathbf{Z}_{\mathbf{0}}$ is

$$
E \exp \left(i \boldsymbol{\omega}^{\prime} \mathbf{Z}_{\mathbf{0}}\right)=\exp \left(-\frac{1}{2} \boldsymbol{\omega}^{\prime} \boldsymbol{\Sigma} \boldsymbol{\omega}\right), \quad \boldsymbol{\omega} \in \mathbb{R}^{d}
$$

From (3.1), we get the characteristic function of $\mathbf{Z}$,

$E \exp \left\{i \boldsymbol{\omega}^{\prime} \mathbf{Z}\right\}$

$$
\begin{aligned}
& =\operatorname{Eexp}\left\{i \boldsymbol{\omega}^{\prime}\left(\lambda U \mathbf{Z}_{0}+\boldsymbol{\mu}\right)\right\} \\
& =\exp \left(i \boldsymbol{\omega}^{\prime} \boldsymbol{\mu}\right) E\left\{\exp \left(i \boldsymbol{\omega}^{\prime} \lambda U \mathbf{Z}_{0}\right)\right\} \\
& =\exp \left(i \boldsymbol{\omega}^{\prime} \boldsymbol{\mu}\right) \int_{0}^{\infty} E\left\{\exp \left(i \boldsymbol{\omega}^{\prime} \lambda U \mathbf{Z}_{0}\right)\right\} f_{U}(u) d u \\
& =\frac{2 \exp \left(i \boldsymbol{\omega}^{\prime} \boldsymbol{\mu}\right)}{\ln \alpha_{1}-\ln \alpha_{2}} \int_{0}^{\infty} u^{-1} \exp \left(-\frac{\lambda^{2} u^{2}}{2} \boldsymbol{\omega}^{\prime} \boldsymbol{\Sigma} \boldsymbol{\omega}\right)\left\{\exp \left(-\alpha_{2} u^{2}\right)-\exp \left(\alpha_{1} u^{2}\right)\right\} d u \\
& =\frac{\exp \left(i \boldsymbol{\omega}^{\prime} \boldsymbol{\mu}\right)}{\ln \alpha_{1}-\ln \alpha_{2}} \int_{0}^{\infty} v^{-1} \exp \left(-\frac{\lambda^{2} v}{2} \boldsymbol{\omega}^{\prime} \boldsymbol{\Sigma} \boldsymbol{\omega}\right)\left\{\exp \left(-\alpha_{2} v\right)-\exp \left(\alpha_{1} v\right)\right\} d v \\
& =\frac{\exp \left(i \boldsymbol{\omega}^{\prime} \boldsymbol{\mu}\right)}{\ln \alpha_{1}-\ln \alpha_{2}}\left[\ln \left(\frac{\lambda^{2}}{2} \boldsymbol{\omega}^{\prime} \boldsymbol{\Sigma} \boldsymbol{\omega}+\alpha_{1}\right)-\ln \left(\frac{\lambda^{2}}{2} \boldsymbol{\omega}^{\prime} \boldsymbol{\Sigma} \boldsymbol{\omega}+\alpha_{2}\right)\right], \quad \boldsymbol{\omega} \in \mathbb{R}^{n},
\end{aligned}
$$

where the last equality follows from the following identity (see Bateman (1954), formula (4), page144), 


$$
\int_{0}^{\infty} w^{-1} \exp (-x w)\left(\exp \left(-\alpha_{2} w\right)-\exp \left(-\alpha_{1} w\right)\right) d w=\ln \left(x+\alpha_{1}\right)-\ln \left(x+\alpha_{2}\right)
$$

In the extreme case where $\alpha_{1}$ tends to $\alpha_{2}$, we obtain the limiting characteristic function as, by using L'Hpital's rule,

$$
\begin{gathered}
\lim _{\alpha_{1} \rightarrow \alpha_{2-}} \frac{\exp \left(i \boldsymbol{\mu}^{\prime} \boldsymbol{\omega}\right)}{\ln \alpha_{1}-\ln \alpha_{2}}\left[\ln \left(\frac{\lambda^{2}}{2} \boldsymbol{\omega}^{\prime} \boldsymbol{\Sigma} \boldsymbol{\omega}+\alpha_{1}\right)-\ln \left(\frac{\lambda^{2}}{2} \boldsymbol{\omega}^{\prime} \boldsymbol{\Sigma} \boldsymbol{\omega}+\alpha_{2}\right)\right] \\
=\frac{\exp \left(i \boldsymbol{\mu}^{\prime} \boldsymbol{\omega}\right)}{1+\frac{\lambda^{2}}{2 \alpha_{2}} \boldsymbol{\omega}^{\prime} \boldsymbol{\Sigma} \boldsymbol{\omega}}, \quad \boldsymbol{\omega} \in \mathbb{R}
\end{gathered}
$$

which is the characteristic function of the double exponential or Laplace random vector.

\subsection{Simulation}

This section is concerned with simulating random numbers from a K-differenced random vector. The simulation from a K-differenced random vector is not straightforward, and it involves simulating random numbers from two independent distributions by using the acceptance-rejection method for the density function in (2.1). As we learned in Section 2.3, it seems appropriate to choose $g(u)$ as a normal density function. In order to be able to reject samples from Normal $(0,1)$ such that they are sampled from $f_{U}(u)$, the normal density must cover the density $f_{U}(u)$. In general, this can be obtained by choosing $c>1$ such that $c g(u)>f_{U}(u)$ for all $u$, where $g(u)$ is the normal density function. The samples are accepted if

$$
\frac{f_{U}(u)}{c g(u)}>v
$$


where $v$ is a random number generated from a uniform distribution over $(0,1)$, and are rejected otherwise. Figure 3.2 gives an visual cognitive about the rejection sample.

Now, let us implement a rejection sampler as a function in the M-file RejectionnonnegativedensityNormal.m (see Appendix B) by using the normal density function which already exists in the MatLab. We generate 2000 random numbers. A histogram for the simulated data in Figure 3.3 looks like a good match with the density (2.1).

In the next step, we need to generate random numbers from a bivariate standard normal distribution, and then use the mixture structure to produce the target data. Hence, the algorithm is

- Sample $U$ from the density function $f_{U}\left(u ; \alpha_{1}, \alpha_{2}\right)$ in (2.1) by using acceptance-rejection method,

- Sample $\mathbf{Z}_{0}$ from a bivariate normal distribution,

- Return $\mathbf{Z}=U \cdot \mathbf{Z}_{0}$.

Applying the above method, we generate 2000 observations from the bi-variate K-differenced distribution with $\Sigma=\left(\begin{array}{cc}1 & 0.5 \\ 0.5 & 1\end{array}\right)$. We then fit the bi-variate K-differenced distribution for the choices of parameters values, e.g. K-differenced $\left(\alpha_{1}=3, \alpha_{2}=12\right)$. Figures 3.4 and 3.5 represent the samples generated by using the algorithm described above.

The same procedure is applied to generate 2000 observation from a bivariate K-differenced distribution with 


$$
\Sigma=\left(\begin{array}{ll}
1 & 0 \\
0 & 1
\end{array}\right)
$$

with the outcomes plotted in Figures 3.7 and 3.8.

\subsection{Maximum likelihood estimation}

For the bivariate K-differenced random vector, forms of the density function and the log-likelihood function exist. For a set of observations, $\left(x_{1}, y_{1}\right), \ldots,\left(x_{n}, y_{n}\right)$, from the $\mathrm{K}$ differenced distribution with parameters $\alpha_{1}, \alpha_{2}$, the log likelihood function of the bi-variate case is

$$
\begin{aligned}
& \ell\left(\alpha_{1}, \alpha_{2}\right)=\sum_{i=1}^{n} \log f\left(y_{i}, x_{i} ; \alpha_{1}, \alpha_{2}\right) \\
&=\sum_{i=1}^{n} \log \left\{\frac { 1 } { \pi \lambda ( \operatorname { l n } \alpha _ { 1 } - \operatorname { l n } \alpha _ { 2 } ) } * \left\{\left(\frac{2 \alpha_{2}}{x^{2}+y^{2}}\right)^{1 / 2} K_{1}\left(\lambda^{-1}\left(2 \alpha_{2}\right)^{1 / 2}\left(x^{2}+y^{2}\right)^{1 / 2}\right)\right.\right. \\
&\left.\left.-\left(\frac{2 \alpha_{1}}{x^{2}+y^{2}}\right)^{1 / 2} K_{1}\left(\lambda^{-1}\left(2 \alpha_{1}\right)^{1 / 2}\left(x^{2}+y^{2}\right)^{1 / 2}\right)\right\}\right\}
\end{aligned}
$$

where $\lambda=\left(\log \alpha_{1}-\log \alpha_{2}\right)^{1 / 2}\left(\alpha_{2}^{-1}-\alpha_{1}^{-1}\right)^{-1 / 2}$. Like the univariate case it must be maximized numerically (using optim in MatLab) to obtain the maximum likelihood estimators.

Now, by using the algorithm mentioned in Section 3.4, we simulate data from the standard bivariate K-differenced distribution with $\alpha_{1}=2, \alpha_{2}=2.5, \boldsymbol{\mu}=\left(\begin{array}{l}0 \\ 0\end{array}\right)$ and 
$\boldsymbol{\Sigma}=\left(\begin{array}{ll}1 & 0 \\ 0 & 1\end{array}\right)$

Applying the simulation method in Section 3.4, we generate 1000 observations from K-differenced with $\alpha_{1}=3$ and $\alpha_{2}=10$. Then we fit the simulated data by finding the maximum likelihood estimators parameters $\alpha_{1}, \alpha_{2}$. Table 3.1 shows a good estimate. 


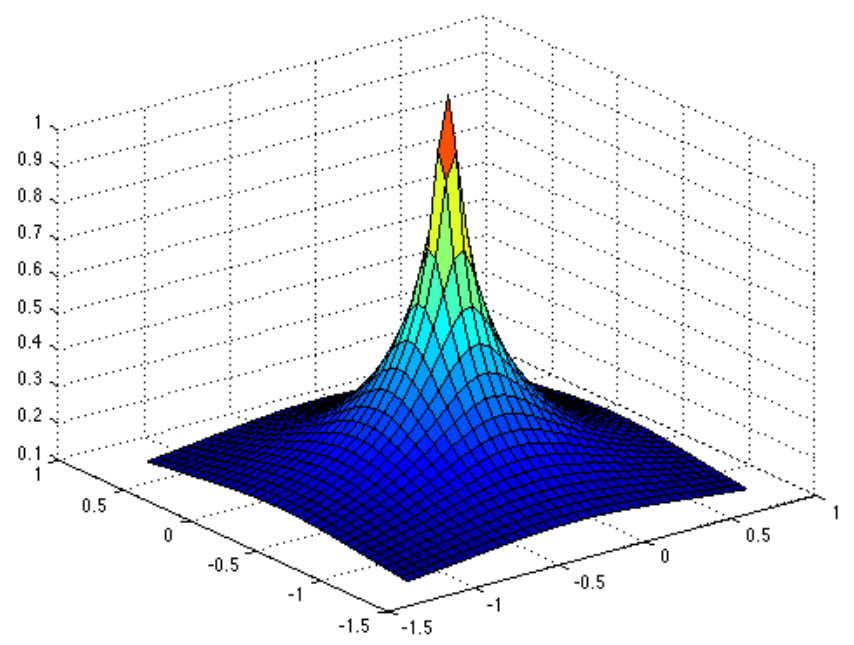

Figure 3.1: Standard bivariate K-differenced density function with $\alpha_{1}=4, \alpha_{2}=5$

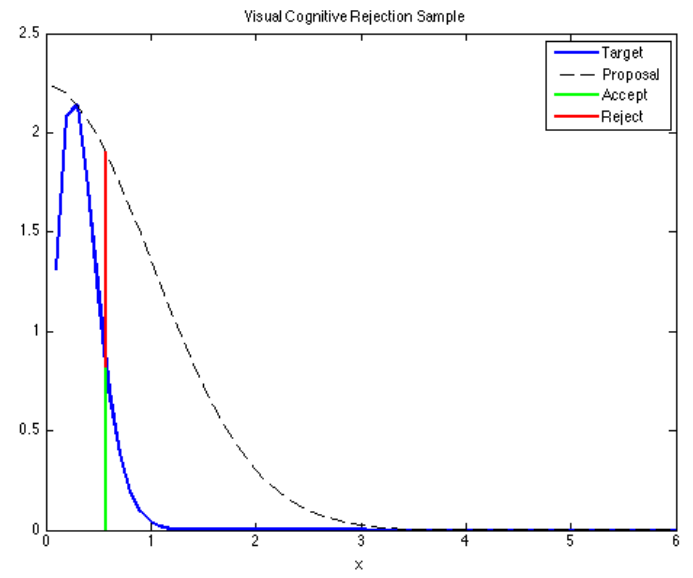

Figure 3.2: Rejection sampling from the density $f_{U}(u)$ based on proposal with $g(u)$ being the standard normal density

Table 3.1: Bivariate K-differenced (using MLE) fitted to the simulated data with $\alpha_{1}=3$ and $\alpha_{2}=10$.

\begin{tabular}{|l|l|l|}
\hline Parameters & $\alpha_{1}$ & $\alpha_{2}$ \\
\hline True value & 3.00 & 10.00 \\
\hline MLE & 3.0375 & 10.1225 \\
\hline
\end{tabular}




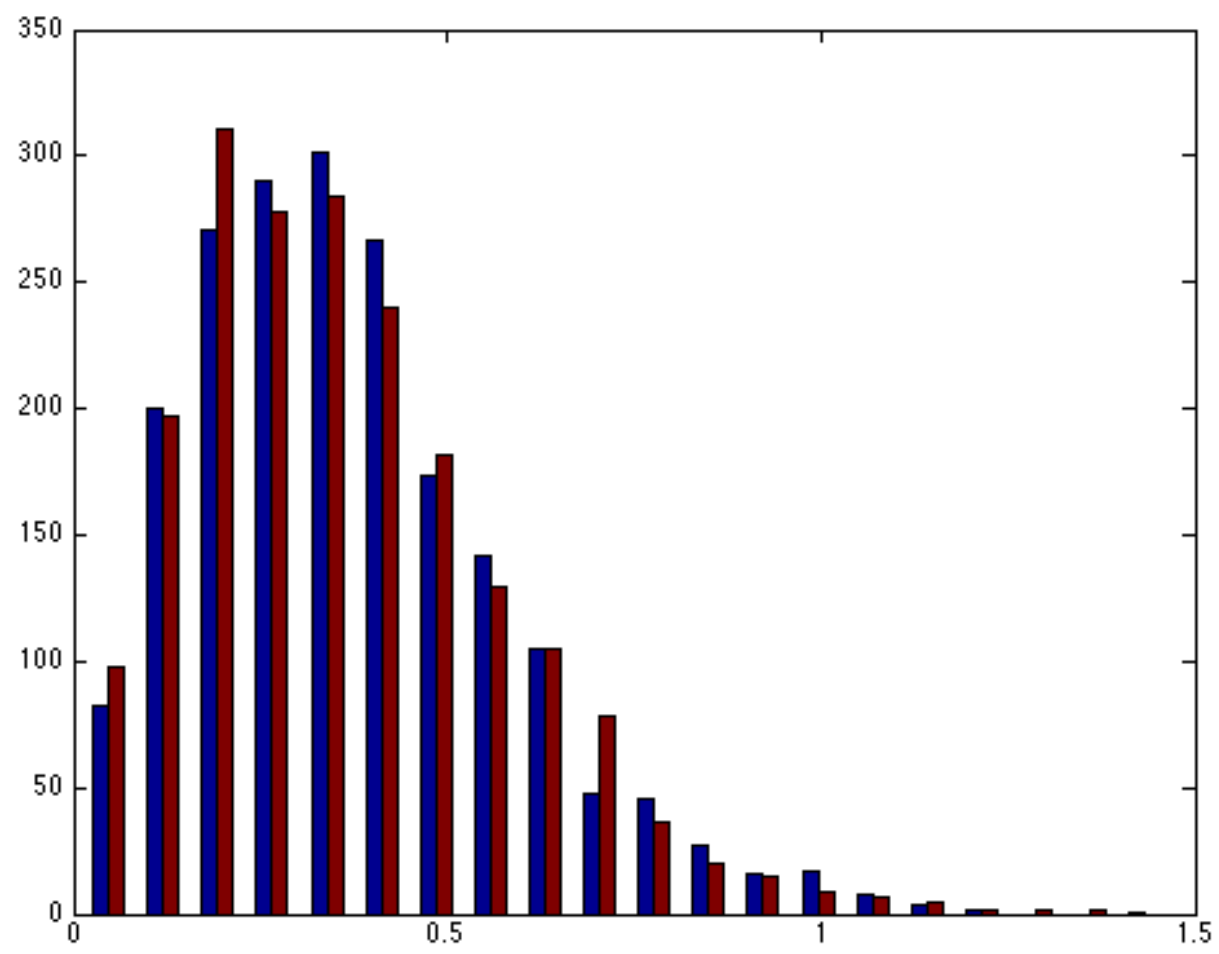

Figure 3.3: Histogram of simulated data from the density $f_{U}(u)$, where $\alpha_{1}=3$ and $\alpha_{2}=12$.

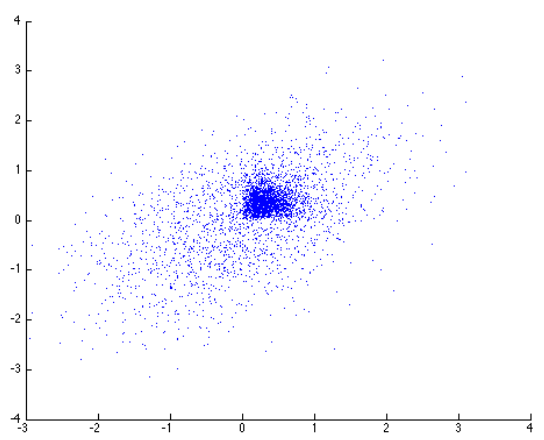

Figure 3.4: Bivariate K-differenced random sample. 


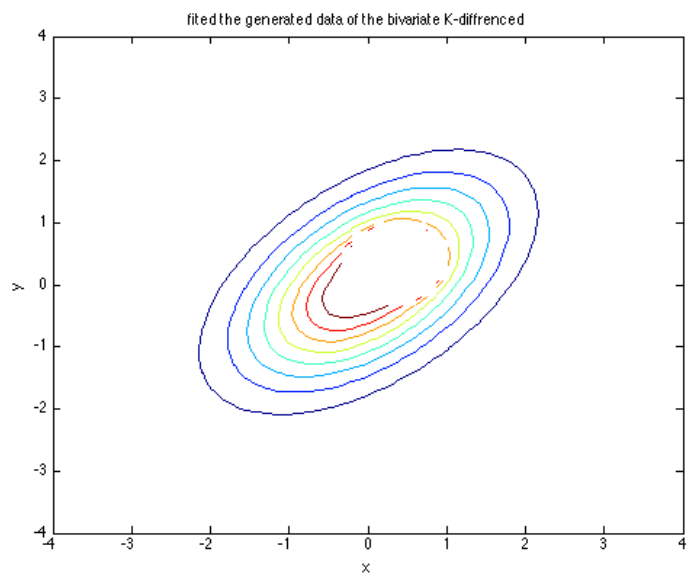

Figure 3.5: Contour plot of bivariate K-differenced sample.

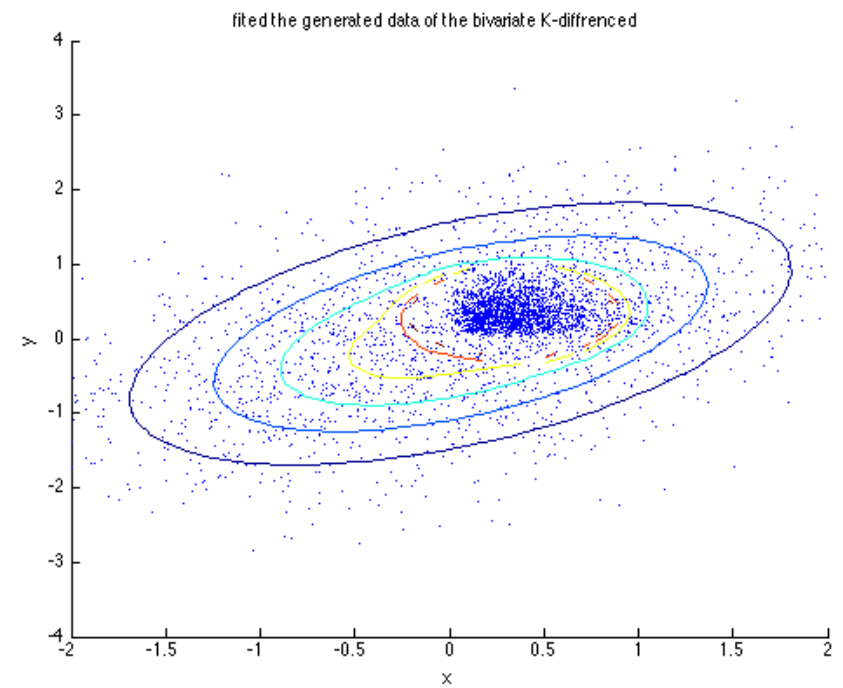

Figure 3.6: Plot of bivariate K-differenced sample. 


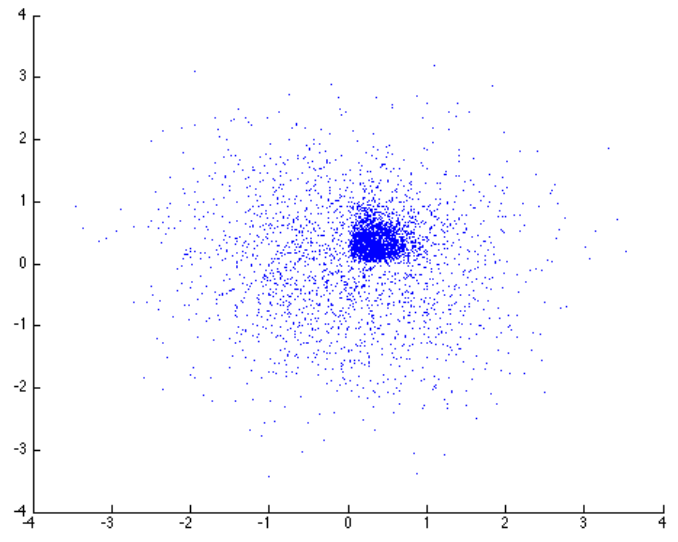

Figure 3.7: Plot of uncorrelated bivariate K-differenced sample.

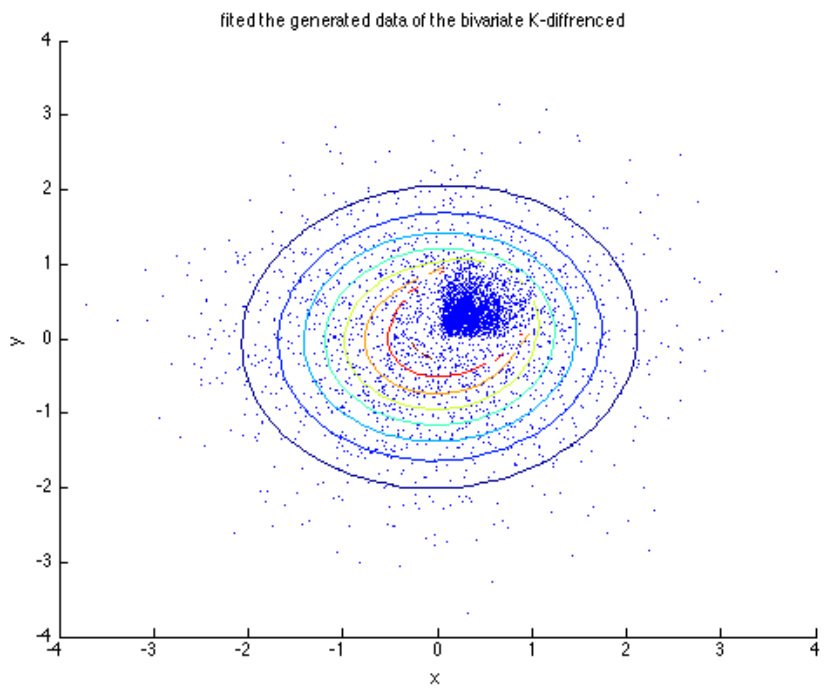

Figure 3.8: Contour plot of uncorrelated bivariate K-differenced sample. 


\section{CHAPTER 4}

\section{K-differenced Vector Random fields}

A K-differenced vector random field is a particular elliptically countered vector random field. This chapter defines a K-differenced vector random field through a stochastic construction in Section 4.1, derives its finite-dimensional characteristic functions in Section 4.2, and discuss its properties in Section 4.3.

\subsection{Stochastic Construction}

Let $\mathbb{D}$ be a temporal, spatial, or spatio-temporal index domain. Suppose that $\left\{\mathbf{Z}_{0}(x), \quad x \in \mathbb{D}\right\}$ is an m-variate Gaussian random field with mean $\mathbf{0}$ and covariance matrix function $\mathbf{C}\left(x_{1}, x_{2}\right)$, $U$ has density (2.1) and is independent with $\left\{\mathbf{Z}_{0}(x), x \in \mathbb{D}\right\}$, and $\boldsymbol{\mu}(x)$ is an m-variate (deterministic) function. We define an m-variate K-differenced random field by

$$
\mathbf{Z}(x)=\lambda U \mathbf{Z}_{0}(x)+\boldsymbol{\mu}(x), \quad x \in \mathbb{D},
$$

where $\lambda=\left(E U^{2}\right)^{-1 / 2}$.

Clearly, $\mathbf{Z}(x)$ follows a K-differenced distribution for each fixed $x \in \mathbb{D}$, with density function

$$
\begin{aligned}
c_{0}\left|\boldsymbol{\Sigma}_{\mathbf{m}}\right|^{-1 / 2}\left\{\left(\frac{\alpha_{2}}{(\mathbf{z}-\boldsymbol{\mu})^{\prime} \mathbf{\Sigma}_{m}^{-1}(\mathbf{z}-\boldsymbol{\mu})}\right)^{m / 4} K_{\frac{m}{2}}\left(\lambda^{-1}\left(2 \alpha_{2}\right)^{1 / 2}\left((\mathbf{z}-\boldsymbol{\mu})^{\prime} \boldsymbol{\Sigma}_{m}^{-1}(\mathbf{z}-\boldsymbol{\mu})\right)^{1 / 2}\right)\right. \\
\left.\quad-\left(\frac{\alpha_{1}}{(\mathbf{z}-\boldsymbol{\mu})^{\prime} \mathbf{\Sigma}_{m}^{-1}(\mathbf{z}-\boldsymbol{\mu})}\right)^{m / 4} K_{\frac{m}{2}}\left(\lambda^{-1}\left(2 \alpha_{1}\right)^{1 / 2}\left((\mathbf{z}-\boldsymbol{\mu})^{\prime} \boldsymbol{\Sigma}_{m}^{-1}(\mathbf{z}-\boldsymbol{\mu})\right)^{1 / 2}\right)\right\}
\end{aligned}
$$

where

$$
c_{0}=\frac{2^{1-m / 4} \pi^{-m / 2} \lambda^{-m / 2}}{\ln \alpha_{1}-\ln \alpha_{2}},
$$


and $\boldsymbol{\Sigma}_{m}=\operatorname{cov}(\mathbf{Z}(x), \mathbf{Z}(x))$. A double exponential distribution is obtained in the extreme case when letting $\alpha_{1}$ tends to $\alpha_{2}$.

\subsection{Finite-dimensional characteristic functions}

We are going to find the finite-dimensional characteristic functions. For every natural number $n$ and any $x_{k} \in \mathbb{D}(\mathrm{k}=1, \ldots, \mathrm{n})$, the characteristic function of an mn-variate random vector $\left(\mathbf{Z}^{\prime}\left(x_{1}\right), \mathbf{Z}^{\prime}\left(x_{2}\right), \ldots, \mathbf{Z}^{\prime}\left(x_{n}\right)\right)^{\prime}$ is

$$
\begin{aligned}
& E \exp \left\{\imath \sum_{k=1}^{n} \boldsymbol{\omega}_{k}^{\prime} \mathbf{Z}\left(x_{k}\right)\right\} \\
& =\operatorname{Eexp}\left\{\imath \sum_{k=1}^{n} \boldsymbol{\omega}_{k}^{\prime}\left(\lambda U \mathbf{Z}_{0}\left(x_{k}\right)+\boldsymbol{\mu}\left(x_{k}\right)\right)\right\} \\
& =\exp \left(\imath \sum_{k=1}^{n} \boldsymbol{\omega}_{k}^{\prime} \boldsymbol{\mu}\left(x_{k}\right)\right) \cdot E\left\{\exp \left(\imath \sum_{k=1}^{n} \lambda U \boldsymbol{\omega}_{k}^{\prime} \mathbf{Z}_{0}\left(x_{k}\right)\right)\right\} \\
& =\exp \left(\imath \sum_{k=1}^{n} \boldsymbol{\omega}_{k}^{\prime} \boldsymbol{\mu}\left(x_{k}\right)\right) \int_{0}^{\infty} E\left\{\exp \left(\imath \lambda U \sum_{k=1}^{n} \boldsymbol{\omega}_{k}^{\prime} \mathbf{Z}_{0}\left(x_{k}\right)\right)\right\} d F_{U}(u) \\
& =\frac{2 \exp \left(\imath \sum_{k=1}^{n} \boldsymbol{\omega}_{k}^{\prime} \boldsymbol{\mu}\left(x_{k}\right)\right)}{\ln \alpha_{1}-\ln \alpha_{2}} \int_{0}^{\infty} u^{-1} \exp \left(-\frac{\lambda^{2} u^{2}}{2} \sum_{i=1}^{n} \sum_{j=1}^{n} \boldsymbol{\omega}_{i}^{\prime} \mathbf{C}\left(x_{i}, x_{j}\right) \boldsymbol{\omega}_{j}\right)\left\{\exp \left(-\alpha_{2} u^{2}\right)-\exp \left(\alpha_{1} u^{2}\right)\right\} d u \\
& =\frac{\exp \left(\imath \sum_{k=1}^{n} \boldsymbol{\omega}_{k}^{\prime} \boldsymbol{\mu}\left(x_{k}\right)\right)}{\ln \alpha_{1}-\ln \alpha_{2}} \int_{0}^{\infty} v^{-1} \exp \left(-\frac{\lambda^{2} v}{2} \sum_{i=1}^{n} \sum_{j=1}^{n} \boldsymbol{\omega}_{i}^{\prime} \mathbf{C}\left(x_{i}, x_{j}\right) \boldsymbol{\omega}_{j}\right)\left\{\exp \left(-\alpha_{2} v\right)-\exp \left(\alpha_{1} v\right)\right\} d v \\
& =\frac{\exp \left(\imath \sum_{k=1}^{n} \boldsymbol{\omega}_{k}^{\prime} \boldsymbol{\mu}\left(x_{k}\right)\right)}{\ln \alpha_{1}-\ln \alpha_{2}}\left[\ln \left(\frac{\lambda^{2}}{2} \sum_{i=1}^{n} \sum_{j=1}^{n} \boldsymbol{\omega}_{i}^{\prime} \mathbf{C}\left(x_{i}, x_{j}\right) \boldsymbol{\omega}_{j}+\alpha_{1}\right)-\ln \left(\frac{\lambda^{2}}{2} \sum_{i=1}^{n} \sum_{j=1}^{n} \boldsymbol{\omega}_{i}^{\prime} \mathbf{C}\left(x_{i}, x_{j}\right) \boldsymbol{\omega}_{j}+\alpha_{2}\right)\right], \\
& \boldsymbol{\omega}_{1}, \ldots ., \boldsymbol{\omega}_{n} \in \mathbb{R}^{m},
\end{aligned}
$$

where the last equality follows from the following identity (see Bateman (1954), formula (4), 
page 144),

$$
\int_{0}^{\infty} t^{-1}(\exp (-\alpha t)-\exp (-\beta t)) \exp (-u t) d t=\ln (u+\beta)-\ln (u+\alpha), \quad u \geq 0 .
$$

In an extreme case where $\alpha_{1}$ tends to $\alpha_{2}$, we obtain the limiting the characteristic function of $\left(\mathbf{Z}^{\prime}\left(x_{1}\right), \mathbf{Z}^{\prime}\left(x_{2}\right), \ldots, \mathbf{Z}^{\prime}\left(x_{n}\right)\right)^{\prime}$ as

$$
\begin{aligned}
& \lim _{\alpha_{1} \rightarrow \alpha_{2-}} E \exp \left\{\imath \sum_{k=1}^{n} \boldsymbol{\omega}_{k}^{\prime} \mathbf{Z}\left(x_{k}\right)\right\} \\
& =\lim _{\alpha_{1} \rightarrow \alpha_{2-}} \frac{\exp \left(\sum_{k=1}^{n} \boldsymbol{\omega}_{k}^{\prime} \boldsymbol{\mu}\left(x_{k}\right)\right)}{\ln \alpha_{1}-\ln \alpha_{2}}\left[\ln \left(\frac{\lambda^{2}}{2} \sum_{i=1}^{n} \sum_{j=1}^{n} \boldsymbol{\omega}_{i}^{\prime} \mathbf{C}\left(x_{i}, x_{j}\right) \boldsymbol{\omega}_{j}+\alpha_{1}\right)\right. \\
& \left.-\ln \left(\frac{\lambda^{2}}{2} \sum_{i=1}^{n} \sum_{j=1}^{n} \boldsymbol{\omega}_{i}^{\prime} \mathbf{C}\left(x_{i}, x_{j}\right) \boldsymbol{\omega}_{j}+\alpha_{2}\right)\right] \\
& =\lim _{\alpha_{1} \rightarrow \alpha_{2}} \exp \left(\imath \sum_{k=1}^{n} \boldsymbol{\omega}_{k}^{\prime} \boldsymbol{\mu}\left(x_{k}\right)\right)\left\{\frac{\frac{\frac{\lambda^{2}}{2} \sum_{i=1}^{n} \sum_{j=1}^{n} \omega_{i}^{\prime} \mathrm{C}\left(x_{i}, x_{j}\right) \omega_{j}+\alpha_{1}}{\frac{1}{\alpha_{1}}}}{\alpha_{1}}\right\} \\
& =\frac{\exp \left(\imath \sum_{k=1}^{n} \boldsymbol{\omega}_{k}^{\prime} \boldsymbol{\mu}\left(x_{k}\right)\right)}{1+\frac{\lambda^{2}}{2 \alpha_{2}} \sum_{i=1}^{n} \sum_{j=1}^{n} \boldsymbol{\omega}_{i}^{\prime} \mathbf{C}\left(x_{i}, x_{j}\right) \boldsymbol{\omega}_{j}}, \quad \boldsymbol{\omega} \in \mathbb{R}^{m},
\end{aligned}
$$

which is the characteristic function of the double exponential or Laplace vector random field.

\subsection{Properties}

The mean and variance functions of $\mathbf{Z}(x)$ defined by (4.1) exist, with

$$
E \mathbf{Z}(x)=\lambda E U E \mathbf{Z}_{0}(x)=\boldsymbol{\mu}(x),
$$

and

$$
\operatorname{cov}(\mathbf{Z}(\mathbf{x}), \mathbf{Z}(\mathbf{x}))
$$




$$
\begin{aligned}
& =\lambda^{2} E U^{2} \operatorname{cov}\left(\mathbf{Z}_{0}(x), \mathbf{Z}_{0}(x)\right) \\
& =\operatorname{cov}\left(\mathbf{Z}_{0}(x), \mathbf{Z}_{0}(x)\right)=\mathbf{C}(x, x), x \in \mathbb{D} .
\end{aligned}
$$

The random field $\{\mathbf{Z}(x), x \in \mathbb{D}\}$ is of second-order, with covariance matrix function

$$
\begin{aligned}
& \operatorname{cov}\left(\mathbf{Z}\left(x_{1}\right), \mathbf{Z}\left(x_{2}\right)\right) \\
& =\operatorname{cov}\left(\lambda U \mathbf{Z}_{0}\left(x_{1}\right)+\boldsymbol{\mu}\left(x_{1}\right), \lambda U \mathbf{Z}_{\mathbf{0}}\left(x_{2}\right)+\boldsymbol{\mu}\left(x_{2}\right)\right) \\
& =\lambda^{2} E U^{2} \operatorname{cov}\left(\mathbf{Z}_{0}\left(x_{1}\right), \mathbf{Z}_{0}\left(x_{2}\right)\right) \\
& =\operatorname{cov}\left(\mathbf{Z}_{0}\left(x_{1}\right), \mathbf{Z}_{0}\left(x_{2}\right)\right), \quad x_{1}, x_{2} \in \mathbb{D} .
\end{aligned}
$$

Hence, $\{\mathbf{Z}(x), x \in \mathbb{D}\}$ and $\left\{\mathbf{Z}_{0}(x), x \in \mathbb{D}\right\}$ have the same covariance matrix functions. This implies that the K-differenced vector random field allows any given correlation structure.

Theorem 1. If $\mathbf{C}\left(x_{1}, x_{2}\right)$ is the covariance matrix function of an m-variate K-differenced random field on $\mathbb{D}$, then

$$
\left(\mathbf{C}\left(x_{1}, x_{2}\right)\right)^{\prime}=\mathbf{C}\left(x_{2}, x_{1}\right),
$$

and the inequality

$$
\sum_{i=1}^{n} \sum_{j=1}^{n} \mathbf{a}_{i}^{\prime} \mathbf{C}\left(x_{i}, x_{j}\right) \mathbf{a}_{j} \geq 0
$$

holds for every natural number $n$, any $x_{i} \in \mathbb{D}$, and any $\mathbf{a}_{i} \in \mathbb{R}^{m}, i=1, \ldots, n$. 
Conversely, for a given $m \times m$ matrix function $\mathbf{C}\left(x_{1}, x_{2}\right)$ that satisfies equation (4.2) and inequality (4.3), an m-variate K-differenced vector random field of the form (4.1) can be defined, with $\mathbf{C}\left(x_{1}, x_{2}\right)$ as its covariance matrix function.

Proof:

If $\mathbf{C}\left(x_{1}, x_{2}\right)$ is the covariance matrix function of $\{\mathbf{Z}(x), x \in \mathbb{D}\}$, then by Theorem 8 of Ma (2011a), equation (4.2) and inequality (4.3) hold.

Conversely, let $\mathbf{C}\left(x_{1}, x_{2}\right)$ be an $m \times m$ matrix function that satisfies equation (4.2) and inequality (4.3), then there exists an m-variate Gaussian random field, $\left\{\mathbf{Z}_{0}(x), x \in \mathbb{D}\right\}$, with mean $\mathbf{0}$ and covariance matrix function $\mathbf{C}\left(x_{1}, x_{2}\right)$. Suppose that $U$ is a random variable with density (2.1) and is independent with $\left\{\mathbf{Z}_{0}(x), x \in \mathbb{D}\right\}$. Then the m-variate K-differenced random field $\{\mathbf{Z}(x), x \in \mathbb{D}\}$ defined by (4.1), with $\lambda=\left(\ln \left(\alpha_{1}\right)-\ln \left(\alpha_{2}\right)\right)^{1 / 2}\left(\alpha_{2}^{-1}-\alpha_{1}^{-1}\right)^{-1 / 2}$, is a random field with covariance matrix function $\mathbf{C}\left(x_{1}, x_{2}\right)$. 


\section{CHAPTER 5}

\section{Some Covariance Matrix Structures}

In Sections 5.2 and 5.3 we present some types of covariance matrix structures for Kdifferenced vector random fields in term of a scalar variogram, a completely monotone function, and a conditionally negative definite matrix, which are reviewed in Section 5.1.

\subsection{Some notations and facts}

An important assumption in our construction of covariance matrix functions is that of conditionally negative definite matrix with non-negative entries. For $m \geq 2$, an $m \times m$ symmetric matrix $\Theta=\left(\theta_{i j}\right)$ is said to be conditionally negative definite, if the inequality

$$
\sum_{i=1}^{m} \sum_{j=1}^{m} \theta_{i j} a_{i} a_{j} \leq 0
$$

holds for any real numbers $a_{1}, \ldots, a_{m}$, subject to the restriction $\sum_{i=1}^{m} a_{i}=0$. If the above inequality holds without a restriction, then $\Theta$ is negative definite. It is known that $\Theta=\left(\theta_{i j}\right)$ is conditionally negative definite if and only if an $m \times m$ matrix with entries $\exp \left(-u \theta_{i j}\right)$ is positive definite for every $u \geq 0$; see Bapat and Reghaven (1997) for more details.

Examples of conditionally negative definite matrices $\Theta=\left(\theta_{i j}\right)$ are

(i) $\theta_{i j}=\theta_{i}+\theta_{j}$,

(ii) $\theta_{i j}=\left|\theta_{i}-\theta_{j}\right|$,

(iii) $\theta_{i j}=\left(\theta_{i}-\theta_{j}\right)^{2}$,

(iv) $\theta_{i j}=-\theta_{i} \theta_{j}$ 
(v) $\theta_{i j}=\max \left(\theta_{i}, \theta_{j}\right), i, j=1, \ldots, m$, where $\theta$ 's are real numbers.

A scalar variogram is an important measure for a scalar random field with second-order increments (see, i.e., Chiles and Delfiner (1999), Cress (1993)). For a scalar random field $\{Z(x), x \in \mathbb{D}\}$ with second order increments, its variograms is defined by

$$
\gamma\left(x_{1}, x_{2}\right)=\frac{1}{2} \operatorname{var}\left(Z\left(x_{1}\right), Z\left(x_{2}\right)\right), x_{1}, x_{2} \in \mathbb{D} .
$$

This function has the following properties

(i) $\gamma\left(x_{1}, x_{2}\right)=\gamma\left(x_{2}, x_{1}\right), x_{1}, x_{2} \in \mathbb{D}$,

(ii) $\gamma(x, x)=0, x \in \mathbb{D}$,

(iii) for every natural number $n \geq 2$, any $x_{i} \in \mathbb{D}$, an $n \times n$ matrix with entries $\gamma\left(x_{i}, x_{j}\right), i, j=$ $1, \ldots, n$, is conditionally negative definite.

According to Schoenberg's theorem (see, e.g., Ma (2005)), a nonnegative function $\gamma\left(x_{1}, x_{2}\right)$ with $\gamma(x, x) \equiv 0$ is a variogram if and only if $\exp \left\{-\gamma\left(x_{1}, x_{2}\right) w\right\}, x_{1}, x_{2} \in \mathbb{D}$, is a scalar covariance function for an arbitrary $w \geq 0$. Hence, an $m \times m$ matrix function with entries all identical to $\exp \left\{-\gamma\left(x_{1}, x_{2}\right) w\right\}$ is a covariance matrix function for each fixed $w \geq 0$.

Example of scalar varigrams $\gamma\left(x_{1}, x_{2}\right)$ are

(i) $\gamma\left(\mathbf{x}_{1}, \mathbf{x}_{2}\right)=\left\|\mathbf{x}_{1}-\mathbf{x}_{2}\right\|^{\lambda}, 0<\lambda \leq 2, \mathbf{x}_{1}, \mathbf{x}_{2} \in \mathbb{R}^{d}$,

(ii) $\gamma\left(\mathbf{x}_{1}, \mathbf{x}_{2}\right)=\left|\mathbf{x}_{1}-\mathbf{x}_{2}\right|, \mathbf{x}_{1}, \mathbf{x}_{2} \in \mathbb{R}^{d}$,

(iii) $\gamma\left(\mathbf{x}_{1}, \mathbf{x}_{2}\right)=\ln \left(1+\left\|\mathbf{x}_{1}-\mathbf{x}_{2}\right\|\right), \quad \mathbf{x}_{1}, \mathbf{x}_{2} \in \mathbb{R}^{d}$, where $\|\mathbf{x}\|$ is the $\ell_{2}$-norm of $\mathbf{x} \in \mathbb{R}^{d}$, and $|\mathbf{x}|$ is the $\ell_{1}$-norm of $\mathbf{x} \in \mathbb{R}^{d}$. 
A non-negative and continuous function $\ell(x), x \geq 0$, is completely monotone on $[0, \infty)$, if it has derivatives of all orders on $(0, \infty)$ and the derivatives alternate in sign, i.e., for every natural number $n,(-1)^{n} \frac{d^{n}}{d x^{n}} \ell(x) \geq 0, x>0$.

Bernstein's theorem asserts that $\ell(x)$ is completely monotone on $[0, \infty)$ if and only if $\ell(x)=\int_{0}^{\infty} \exp (-x u) d \mu(u)$, where $\mu(u)$ is non-negative finite measure on $[0, \infty)$. Clearly, the Laplace transform, whenever it exists, of a non-negative random variable is a completely monotone function on $[0, \infty)$.

Example of completely monotone functions $\ell(x)$, on $[0, \infty)$ are

(i) $\ell(x)=(x+2 \alpha) \ln (x+2 \alpha)+x \ln (x)-2(x+\alpha) \ln (x+\alpha)$, with $\alpha>0$,

(ii) $\ell(x)=(1+\exp (x))^{-1}$,

(iii) $\ell(x)=\beta^{\frac{1}{2}} \operatorname{Erfc}\left(\beta^{1 / 2} x^{1 / 2}\right)$, with $\beta>0$.

\subsection{Covariance matrix structure I}

In this section we are going to present some types of covariance matrix structures with three ingredients: a completely monotone function, a scalar variogram, and a conditionally negative definite matrix.

Theorem 2: Assume that $\ell(x)$ is a completely monotone function on $[0, \infty)$. If $\gamma\left(x_{1}, x_{2}\right)$ is a scalar variogram on $\mathbb{D}$, and $\Theta=\left(\theta_{i j}\right)$ is an $m \times m$ conditionally negative definite matrix with nonnegative entries, then there exists an m-variate $\mathrm{K}$-differenced random field on $\mathbb{D}$, with direct and cross covariances

$$
C_{i j}\left(x_{1}, x_{2}\right)=\ell\left(\gamma\left(x_{1}, x_{2}\right)+\theta_{i j}\right), \quad x_{1}, x_{2} \in \mathbb{D}, \quad i, j=1, \ldots m
$$


Proof:

By Bernstein's theorem (see Schilling, R. et al. (2010)), the completely monotone function $\ell(x)$ is the Laplace transform of a bounded, non-decreasing function $F(u), u \geq 0$; that is

$$
\ell(x)=\int_{0}^{\infty} \exp (-x u) d F(u), \quad x \geq 0 .
$$

With this representation, the functions in (5.1) are the same as

$$
\begin{aligned}
C_{i j}\left(x_{1}, x_{2}\right) & =\int_{0}^{\infty} \exp \left(-\left(\gamma\left(x_{1}, x_{2}\right)+\theta_{i j}\right) u\right) d F(u) \\
& =\int_{0}^{\infty} \exp \left(-\gamma\left(x_{1}, x_{2}\right) u\right) \exp \left(-\theta_{i j} u\right) d F(u) \\
& =\int_{0}^{\infty} C_{i j}\left(x_{1}, x_{2} ; u\right) d F(u), \quad x_{1}, x_{2} \in \mathbb{D}, i, j=1, \ldots, m,
\end{aligned}
$$

where

$$
C_{i j}\left(x_{1}, x_{2} ; u\right)=\exp \left(-\gamma\left(x_{1}, x_{2}\right) u\right) \exp \left(-\theta_{i j} u\right), x_{1}, x_{2} \in \mathbb{D}, u \geq 0 .
$$

Thus it suffices to show that for every fixed $u \geq 0, C_{i j}\left(x_{1}, x_{2} ; u\right)$ are direct/cross covariances in $\mathbb{D}$.

Since $\gamma\left(x_{1}, x_{2}\right)$ is a scalar variogram, $\exp \left(-\gamma\left(x_{1}, x_{2}\right) u\right), x_{1}, x_{2} \in \mathbb{D}$, is a covariance function for every fixed $u \geq 0$, by Schoenberg's theorem (see, e.g., Ma (2005)). An $m \times m$ matrix with entries $\exp \left(-\theta_{i j} u\right)$ is positive definite, since $\Theta$ is conditionally negative definite. Thus, an $m \times m$ matrix function with entries $C_{i j}\left(x_{1}, x_{2} ; u\right)=\exp \left(-\gamma\left(x_{1}, x_{2}\right) u\right) \exp \left(-\theta_{i j} u\right), x_{1}, x_{2} \in$ $\mathbb{D}$, is a covariance matrix function. Hence, by Theorem 4 of Ma (2011c), the $m \times m$ matrix 
function with entries $C_{i j}\left(x_{1}, x_{2} ; u\right)$ is a covariance matrix function.

Obviously, a K-differenced vector random field with direct/cross covariances (5.1) is generally not stationary, unless $\gamma\left(x_{1}, x_{2}\right)$ is intrinsically stationary, in the sense that it is a function of $x_{1}-x_{2}$ only. Many examples of (5.1) can be obtained once $\gamma\left(x_{1}, x_{2}\right)$ and $\ell(x)$ are specified.

Example 1. For $0<\lambda \leq 2$, in (5.1) letting $\gamma\left(\mathbf{x}_{1}, \mathbf{x}_{2}\right)=\left\|\mathbf{x}_{1}-\mathbf{x}_{2}\right\|^{\lambda}$ yields

$$
C_{i j}\left(x_{1}, x_{2}\right)=\ell\left(\left\|\mathbf{x}_{1}-\mathbf{x}_{2}\right\|^{\lambda}+\theta_{i j}\right), \mathbf{x}_{1}, \mathbf{x}_{2} \in \mathbb{R}^{d}, i, j=1, \ldots, m,
$$

which are the direct and cross covariances of a stationary m-variate random field in $\mathbb{D}$.

$$
\begin{aligned}
& \text { Example 2. In (5.1) choosing } \ell(x)=\beta^{-1 / 2} \operatorname{Erfc}\left(\beta^{1 / 2} x^{1 / 2}\right) \text { yields } \\
& C_{i j}\left(x_{1}, x_{2}\right)=\beta^{-1 / 2} \operatorname{Erfc}\left(\beta^{1 / 2}\left(\gamma\left(x_{1}, x_{2}\right)+\theta_{i j}\right)^{1 / 2}\right)
\end{aligned}
$$

$$
x_{1}, x_{2} \in \mathbb{D}, i, j=1, \ldots m,
$$

where $\beta>0$. Here $\ell(x)$ is complete monotone on $[0, \infty)$, since it can be rewritten via the identity (see Bateman (1954), formula 26, p.136).

$$
(\pi)^{-1} \int_{\beta}^{\infty} \omega^{-1}(\omega-\beta)^{-1 / 2} \exp (-x \omega) d \omega=\beta^{-1 / 2} \operatorname{Erfc}\left(\beta^{1 / 2} x^{1 / 2}\right), \quad \mathbf{x}_{\iota} 0 .
$$


It is known that $\|\mathbf{x}\|$ and $|\mathbf{x}|, \mathbf{x} \in \mathbb{R}^{d}$, are intrinsically stationary variograms (see Cressie (1993), Chiles and Delfiner (1999)). Two covariance matrix functions are constructed in Theorems 3 and 4, respectively, with the following three ingredients: the Euclidean norm $\|\mathbf{x}\|$ or $\ell_{1}$-norm $|\mathbf{x}|$, a conditionally negative definite matrix $\Theta$, and a completely monotonic function $\ell(x)$.

Theorem 3. Assume that $\ell(x)$ is a completely monotone function on $[0, \infty)$. If $\Theta=\left(\theta_{i j}\right)$ is an $m \times m$ conditionally negative definite matrix with positive entries, then there exists an $\mathrm{m}$-variate stationary K-differenced random field in $\mathbb{R}^{d}$, with direct and cross covariances

$$
C_{i j}(\mathbf{x})=\theta_{i j}^{-\frac{d}{2}} \ell\left(\frac{\|\mathbf{x}\|^{2}}{\theta_{i j}}\right), \quad \mathbf{x} \in \mathbb{R}^{d}, i, j=1, \ldots, m
$$

Proof:

With the representation (5.2) of $\ell(x)$ on $[0, \infty)$, we rewrite the functions in (5.4) as

$$
\begin{aligned}
& C_{i j}(\mathbf{x}) \\
& =\theta_{i j}^{-\frac{d}{2}} \int_{0}^{\infty} \exp \left(-\frac{\|\mathbf{x}\|^{2}}{\theta_{i j}} u\right) d F(u) \\
& =\int_{0}^{\infty} C_{i j}(\mathbf{x} ; u) d F(u),
\end{aligned}
$$

where

$$
C_{i j}(\mathbf{x} ; u)=\theta_{i j}^{-\frac{d}{2}} \exp \left(-\frac{\|\mathbf{x}\|^{2}}{\theta_{i j}} u\right), \quad \mathbf{x} \in \mathbb{R}^{d}, i, j=1, \ldots, m
$$


Thus it suffices to show that for every fixed $u \geq 0, C_{i j}(\mathbf{x} ; u)(i, j=1, \ldots, m)$ are direct and cross covariances in $\mathbb{R}^{d}$.

Notice that the following identity holds for a strictly positive definite matrix $\mathbf{A}$,

$$
|\mathbf{A}|^{-\frac{1}{2}} \exp \left(-\frac{1}{2} \mathbf{x}^{\prime} \mathbf{A}^{-1} \mathbf{x}\right)=(2 \pi)^{-\frac{d}{2}} \int_{\mathbb{R}^{d}} \exp \left(\imath \omega^{\prime} \mathbf{x}-\frac{1}{2} \omega^{\prime} \mathbf{A} \omega\right) d \omega, \quad \mathbf{x} \in \mathbb{R}^{d}
$$

Replacing $\mathbf{A}$ by $\theta_{i j} \mathbf{I}_{d}$ and $\mathbf{x}$ by $\mathbf{x} \sqrt{2 u}$, where $\mathbf{I}_{d}$ is a $d \times d$ identity matrix, we rewrite $C_{i j}(\mathbf{x} ; u)$ as

$$
\begin{aligned}
& C_{i j}(\mathbf{x} ; u)=(2 \pi)^{-\frac{d}{2}} \int_{\mathbb{R}^{d}} \exp \left(\imath \omega^{\prime} \mathbf{x} \sqrt{2 u}-\frac{1}{2} \theta_{i j}\|\omega\|^{2}\right) d \omega \\
& =(2 \pi)^{-\frac{d}{2}} \int_{\mathbb{R}^{d}} \cos \left\{\omega^{\prime} \mathbf{x} \sqrt{2 u}\right\} \exp \left(-\frac{1}{2} \theta_{i j}\|\omega\|^{2}\right) d \omega, \quad \mathbf{x} \in \mathbb{R}^{d}, i, j=1, \ldots, m .
\end{aligned}
$$

Indeed this is a covariance matrix function in $\mathbb{R}^{d}$ because for every $\omega \in \mathbb{R}^{d}$,

$$
\cos \left\{\omega^{\prime} \mathbf{x} \sqrt{2 u}\right\}, \mathbf{x} \in \mathbb{R}^{m}
$$

is a scalar stationary covariance function. Also, the $m \times m$ matrix with entries $\exp \left(-\frac{1}{2} \theta_{i j}\|\omega\|^{2}\right)$ is positive definite for each fixed $\omega \in \mathbb{R}^{d}$, due to the assumption that $\Theta$ is conditionally negative definite. Thus, functions in (5.4) form a covariance matrix function.

Example 3. In (5.4) letting

$$
\ell(x)=(x+2 \alpha) \ln (x+2 \alpha)+x \ln (x)-2(x+\alpha) \ln (x+\alpha), \quad x \geq 0,
$$


where $\alpha$ is a positive constant, yields that

$C_{i j}(\mathbf{x})=\theta_{i j}^{-\frac{1}{2}}\left[\left(\frac{\|\mathbf{x}\|^{2}}{\theta_{i j}}+2 \alpha\right) \ln \left(\frac{\|\mathbf{x}\|^{2}}{\theta_{i j}}+2 \alpha\right)+\frac{\|\mathbf{x}\|^{2}}{\theta_{i j}} \ln \left(\frac{\|\mathbf{x}\|^{2}}{\theta_{i j}}\right)-2\left(\frac{\|\mathbf{x}\|^{2}}{\theta_{i j}}+\alpha\right) \ln \left(\frac{\|\mathbf{x}\|^{2}}{\theta_{i j}}+\alpha\right)\right]$,

$$
\mathbf{x} \in \mathbb{R}^{d}, i, j=1, \ldots, m
$$

form the direct and cross covariances of an m-variate K-differenced random field, since $\ell(x)$ can be written as (see Bateman (1954), formula 5, p.144)

$$
\int_{0}^{\infty} \omega^{-2}(1-\exp (-\alpha \omega))^{2} \exp (-x \omega) d \omega=(x+2 \alpha) \ln (x+2 \alpha)+x \ln (x)-2(x+\alpha) \ln (x+\alpha) .
$$

Theorem 4: Assume $\ell(x)$ is a completely monotone function on $[0, \infty)$. If $\Theta=\left(\theta_{i j}\right)$ is an $m \times m$ conditionally negative definite matrix with positive entries, then there exists an $\mathrm{m}$-variate stationary $\mathrm{K}$-differenced random field in $\mathbb{R}^{d}$, with direct and cross covariances

$$
\begin{aligned}
C_{i j}(\mathbf{x}) & =\theta_{i j}^{-\frac{1}{2}} \ell\left(\theta_{i j}^{\frac{1}{2}}|\mathbf{x}|^{\frac{1}{2}}\right) \\
\mathbf{x} & \in \mathbb{R}^{d}, i, j=1, \ldots, m .
\end{aligned}
$$

Proof:

With the representation of $\ell(x)$ in (5.2), the functions in (5.5) are the same as

$$
\begin{aligned}
& C_{i j}(\mathbf{x})=\theta_{i j}^{-\frac{1}{2}} \int_{0}^{\infty} \exp \left(-\theta_{i j}^{\frac{1}{2}}|\mathbf{x}|^{\frac{1}{2}} u\right) d F(u) \\
& =\int_{0}^{\infty} C_{i j}(\mathbf{x} ; u) \quad d F(u),
\end{aligned}
$$

where 


$$
C_{i j}(\mathbf{x} ; u)=\theta_{i j}^{-\frac{1}{2}} \exp \left(-\theta_{i j}^{\frac{1}{2}}|\mathbf{x}|^{\frac{1}{2}} u\right), \mathbf{x} \in \mathbb{R}^{d}, i, j=1, \ldots, m .
$$

The following identity found in Bateman (1954), formula 5, p.144,

$$
\int_{0}^{\infty} t^{-1 / 2} \exp \left(-\frac{1}{4} \frac{y}{t}\right) \exp (-\beta t) d t=\pi^{1 / 2} \beta^{-1 / 2} \exp \left(-y^{1 / 2} \beta^{1 / 2}\right), \beta>0
$$

Let $y=|\mathbf{x}| u^{2}$ and $\beta=\theta_{i j}$. Then

$$
\begin{aligned}
& \pi^{-1 / 2} \int_{0}^{\infty} t^{-1 / 2} \exp \left(-\frac{1}{4} \frac{|\mathbf{x}| u^{2}}{t}\right) \exp \left(-\theta_{i j} t\right) d t \\
& =\left(\theta_{i j}\right)^{-1 / 2} \exp \left(-\theta_{i j}^{1 / 2}|\mathbf{x}|^{1 / 2} u\right)
\end{aligned}
$$

Since $\Theta=\left(\theta_{i j}\right)$ is conditionally negative definite, the matrix with entries $\exp \left(-\theta_{i j} t\right)$ is pos-

itive definite for every $t \geq 0$. The matrix function $\exp \left(-\frac{1}{4} \frac{|\mathbf{x}| u^{2}}{t}\right) \mathbf{1}$ is obviously a covariance matrix function. Therefore, $C_{i j}(\mathbf{x}, u),(i, j=1, \ldots, m)$ form a covariance matrix function.

Example 4. In (5.5) choosing $\ell(x)=(1+\exp (x))^{-1}, \mathrm{x} \geq 0$, yields that

$$
C_{i j}(\mathbf{x})=\theta_{i j}^{-\frac{1}{2}}\left\{1+\exp \left(\theta_{i j}^{1 / 2}|\mathbf{x}|^{\frac{1}{2}}\right)\right\}^{-1}, \quad \mathbf{x} \in \mathbb{R}^{d}, i, j=1, \ldots, m,
$$

are the direct and cross covariance of a stationary m-variate random field in $\mathbb{R}^{d}$, with long range dependence.

\subsection{Covariance matrix structure II}

In this section we construct some types of covariance matrix structures based on the following theorem, "If $g(\boldsymbol{\omega})$ is nonnegative function in $\mathbb{R}^{d}$ and $\mathbf{C}\left(x_{1}, x_{2} \mid \boldsymbol{\omega}\right)$ is an $m \times m$ 
covariance matrix function on $\mathbb{D}$ for every fixed $\boldsymbol{\omega} \in \mathbb{R}^{d}$, then there is an m-variate secondorder random field with direct and cross covariances

$$
\int_{\mathbb{R}^{d}} \mathbf{C}_{i j}\left(x_{1}, x_{2} \mid \boldsymbol{\omega}\right) g(\boldsymbol{\omega}) d \boldsymbol{\omega}, \quad x_{1}, x_{2} \in \mathbb{D}, \quad i, j=1,2, \ldots, m
$$

assuming that the above integrals exist". See Ma (2011).

Theorem 5. Let $\alpha$ be a positive constant and let $\Theta=\left(\theta_{i j}\right)$ be an $m \times m$ conditionally negative definite matrix with all entries greater than or equal to $\alpha$. If $g_{i j}\left(x_{1}, x_{2}\right)$, $x_{1}, x_{2} \in \mathbb{D}$, are positive functions such that, for each fixed $\omega \geq 0$, an $m \times m$ matrix with entries $\exp \left(-g_{i j}\left(x_{1}, x_{2}\right) \omega\right)$ is a covariance matrix function, then there is an m-variate $\mathrm{K}$ differenced random field on $\mathbb{D}$ with direct and cross covariances

$$
\begin{gathered}
C_{i j}\left(x_{1}, x_{2}\right)=\ln \left(g_{i j}\left(x_{1}, x_{2}\right)+\theta_{i j}+\alpha\right)-\ln \left(g_{i j}\left(x_{1}, x_{2}\right)+\theta_{i j}-\alpha\right), \\
x_{1}, x_{2} \in \mathbb{D}, \quad i, j=1, \ldots, m .
\end{gathered}
$$

Proof:

By using the formula (see Bateman (1954), formula 12, page.163),

$$
\int_{0}^{\infty} 2 \omega^{-1} \sinh (\alpha \omega) \exp (-x \omega) d \omega=\ln (x+\alpha)-\ln (x-\alpha), x>\alpha>0
$$

we obtain

$$
\begin{gathered}
C_{i j}\left(x_{1}, x_{2}\right)=\int_{0}^{\infty} 2 \omega^{-1} \sinh (\alpha \omega) \exp \left(-g_{i j}\left(x_{1}, x_{2}\right) \omega\right) \exp \left(-\theta_{i j} \omega\right) d \omega \\
i, j=1, \ldots, m
\end{gathered}
$$


where $2 \omega^{-1} \sinh (\alpha \omega) \geq 0, \omega \geq 0$. To show that these $m \times m$ functions form the covariance matrix function of an m-variate K-differenced random field, by Theorem 4 of Ma (2011c), we just need verify that the following $m \times m$ functions form a covariance matrix function for each $w>0$,

$$
\begin{gathered}
\widetilde{C}_{i j}\left(x_{1}, x_{2}\right)=\exp \left(-g_{i j}\left(x_{1}, x_{2}\right) \omega\right) \exp \left(-\theta_{i j} \omega\right), \\
x_{1}, x_{2} \in \mathbb{D}, i, j=1, \ldots, m .
\end{gathered}
$$

For a fixed $\omega>0$, an $m \times m$ matrix function with entries $\widetilde{C}_{i j}\left(x_{1}, x_{2}\right)$ is the Hadamard (Schur) product of the following two matrices, the first one with entries

$$
\exp \left(-g_{i j}\left(x_{1}, x_{2}\right) \omega\right), x_{1}, x_{2} \in \mathbb{D}, \quad i, j=1, \ldots, m,
$$

which is a covariance matrix function by assumption, and the second one with entries $\exp \left(-\theta_{i j} \omega\right)$, which is positive definite under the assumption that $\Theta=\left(\theta_{i j}\right)$ is conditionally negative definite (Bapat and Raghaven (1997), Theorem 4.1.3). Hence, $\widetilde{C}_{i j}\left(x_{1}, x_{2}\right)$ is a covariance matrix function, due to Theorem 3 of Ma (2011c).

Example 5. In Theorem 6 letting $g_{i j}\left(\mathbf{x}_{1}, \mathbf{x}_{2}\right)=\left\|\mathbf{x}_{1}-\mathbf{x}_{2}\right\|^{2}$ yields

$$
\begin{gathered}
C_{i j}\left(\mathbf{x}_{1}, \mathbf{x}_{2}\right)=\ln \left(\left\|\mathbf{x}_{1}-\mathbf{x}_{2}\right\|^{2}+\theta_{i j}+\alpha\right)-\ln \left(\left\|\mathbf{x}_{1}-\mathbf{x}_{2}\right\|^{2}+\theta_{i j}-\alpha\right) \\
\mathbf{x}_{1}, \mathbf{x}_{2} \in \mathbb{R}^{d}, \quad i, j=1, \ldots, m,
\end{gathered}
$$

which are the direct and cross covariances of a stationary m-variate K-difference random field in $\mathbb{R}^{d}$ that has long range dependence. 
Theorem 6. Let $\alpha$ be a positive constant. If $g_{i j}\left(x_{1}, x_{2}\right), x_{1}, x_{2} \in \mathbb{D}, \mathrm{i}, \mathrm{j}=1, \ldots, \mathrm{m}$, are positive functions such that, for each fixed $w \geq 0$, an $m \times m$ matrix with entries $\exp \left(-g_{i j}\left(x_{1}, x_{2}\right) w\right)$ is a covariance matrix function, $\Psi\left(x_{1}, x_{2}\right)=\left(\psi_{i j}\left(x_{1}, x_{2}\right)\right)$ is an $m \times m$ covariance matrix function, and $\Theta=\left(\theta_{i j}\right)$ is an $m \times m$ conditionally negative definite matrix with all entries greater than or equal to $\alpha$, then there is an m-variate $\mathrm{K}$-differenced random field on $\mathbb{D}$ with direct and cross covariances

$$
\begin{array}{r}
\mathrm{C}_{i j}\left(x_{1}, x_{2}\right)=\left(\frac{g_{i j}\left(x_{1}, x_{2}\right)+\theta_{i j}+\alpha}{g_{i j}\left(x_{1}, x_{2}\right)+\theta_{i j}-\alpha}\right)^{\psi_{i j}\left(x_{1}, x_{2}\right)} \\
x_{1}, x_{2} \in \mathbb{D}, i, j=1, \ldots, m .
\end{array}
$$

Proof:

In terms of the Taylor expansion of $\exp (x)$, the function $C_{i j}\left(x_{1}, x_{2}\right)$ can be written as

$$
C_{i j}\left(x_{1}, x_{2}\right)=\sum_{n=0}^{\infty} \frac{1}{n !}\left\{\widetilde{C}_{i j}\left(x_{1}, x_{2}\right)\right\}^{n}, \quad x_{1}, x_{2} \in \mathbb{D}, i, j=1, \ldots, m,
$$

where

$$
\widetilde{C}_{i j}\left(x_{1}, x_{2}\right)=\psi_{i j}\left(x_{1}, x_{2}\right)\left[\ln \left(g_{i j}\left(x_{1}, x_{2}\right)+\theta_{i j}+\alpha\right)-\ln \left(g_{i j}\left(x_{1}, x_{2}\right)+\theta_{i j}-\alpha\right)\right] .
$$

By Theorem 5, an $m \times m$ matrix function with entries $\ln \left(g_{i j}\left(x_{1}, x_{2}\right)+\theta_{i j}+\alpha\right)-$ $\ln \left(g_{i j}\left(x_{1}, x_{2}\right)+\theta_{i j}-\alpha\right)$ is a covariance matrix function. By assumption, $\Psi\left(x_{1}, x_{2}\right)$ is a 
covariance matrix function. Hence, the Hadamard product of these two matrix functions, an $m \times m$ matrix function with entries $\widetilde{C}_{i j}\left(x_{1}, x_{2}\right)$ is also a covariance matrix function by Theorem 3 of Ma (2011c). Also, for each $n$, an $m \times m$ matrix function with entries $\left\{\widetilde{C}_{i j}\left(x_{1}, x_{2}\right)\right\}^{n}$ is a covariance matrix function. Hence, an $m \times m$ matrix function with entries $C_{i j}\left(x_{1}, x_{2}\right)$ is also covariance matrix function by Theorems 1 and 2 of Ma (2011c).

The next corollary is obtained from Theorem 6 by taking $g_{i j}\left(x_{1}, x_{2}\right)=g_{i}\left(x_{1}\right)+g_{j}\left(x_{2}\right), x_{1}, x_{2} \in$ $\mathbb{D}, i, j=0, \ldots, m$, since it can be shown that $\exp \left(-\left(g_{i}\left(x_{1}\right)+g_{j}\left(x_{2}\right)\right) w\right)$ is a covariance matrix function, for each fixed $w \geq 0$.

Corollary 1 . With $m$ positive functions $g_{1}(x), \ldots, g_{m}(x), x \in \mathbb{D}$, there is an m-variate K-differenced random field on $\mathbb{D}$ with direct and cross covariances

$$
\begin{gathered}
C_{i, j}\left(x_{1}, x_{2}\right)=\left(\frac{g_{i}\left(x_{1}\right)+g_{j}\left(x_{2}\right)+\theta_{i j}+\alpha}{g_{i}\left(x_{1}\right)+g_{j}\left(x_{2}\right)+\theta_{i j}-\alpha}\right)^{\psi_{i j}\left(x_{1}, x_{2}\right)} \\
x_{1}, x_{2} \in \mathbb{D}, i, j=1, \ldots, m
\end{gathered}
$$

Corollary 2. With $m$ real-valued functions $g_{1}(x), \ldots, g_{m}(x), x \in \mathbb{D}$, there is an m-variate K-differenced random field on $\mathbb{D}$ with direct and cross covariances

$$
\begin{gathered}
C_{i j}\left(x_{1}, x_{2}\right)=\left(\frac{\left|g_{i}\left(x_{1}\right)-g_{j}\left(x_{2}\right)\right|+\theta_{i j}+\alpha}{\left|g_{i}\left(x_{1}\right)-g_{j}\left(x_{2}\right)\right|+\theta_{i j}-\alpha}\right)^{\psi_{i j}\left(x_{1}, x_{2}\right)} \\
x_{1}, x_{2} \in \mathbb{D}, i, j=1, \ldots, m
\end{gathered}
$$

To see this, let $\{Z(x), x \in \mathbb{R}\}$ be an Ornstein-Uhlenbeck process with covariance $\exp \left(-\left|x_{1}-x_{2}\right|\right), x_{1}, x$ $\mathbb{R}$. For each fixed $w \geq 0$,

$$
\left\{\mathbf{Z}(x)=\left(Z\left(g_{1}(x) w\right), \ldots, Z\left(g_{m}(x) w\right)\right)^{\prime}, x \in \mathbb{D}\right\}
$$


is an m-variate random field. Clearly, it has the direct/cross covariances exp $\left(-g_{i j}\left(x_{1}, x_{2}\right) w\right)$, with $g_{i j}\left(x_{1}, x_{2}\right)=\left|g_{i}\left(x_{1}\right)-g_{j}\left(x_{2}\right)\right|, x_{1}, x_{2} \in \mathbb{D}, i, j=1, \ldots, m$.

Theorem 7: If $g_{i j}\left(x_{1}, x_{2}\right), x_{1}, x_{2} \in \mathbb{D}$, are positive functions such that, for each fixed $\omega \geq 0$, an $m \times m$ matrix with entries $\exp \left(-g_{i j}\left(x_{1}, x_{2}\right) \omega\right)$ is a covariance matrix function and $\Theta=\left(\theta_{i j}\right)$ is an $m \times m$ conditionally negative definite matrix with positive entries, then there is an $\mathrm{m}$-variate $\mathrm{K}$-differenced random field on $\mathbb{D}$ with direct and cross covariances

$$
C_{i j}\left(x_{1}, x_{2}\right)=\ell\left(g_{i j}\left(x_{1}, x_{2}\right)+\theta_{i j}\right), \quad x_{1}, x_{2} \in \mathbb{D}, i, j=1, \ldots m .
$$

Proof

With (5.2) representation, the functions in (5.8) are the same as

$$
\begin{aligned}
C_{i j}\left(x_{1}, x_{2}\right) & =\int_{0}^{\infty} \exp \left(-\left(g_{i j}\left(x_{1}, x_{2}\right)+\theta_{i j}\right) u\right) d F(u) \\
& =\int_{0}^{\infty} \exp \left(-g_{i j}\left(x_{1}, x_{2}\right) u\right) \exp \left(-\theta_{i j} u\right) d F(u) \\
& =\int_{0}^{\infty} C_{i j}\left(x_{1}, x_{2} ; u\right) d F(u), \quad x_{1}, x_{2} \in \mathbb{D}, i, j=1, \ldots, m,
\end{aligned}
$$

where

$$
C_{i j}\left(x_{1}, x_{2} ; u\right)=\exp \left(-g_{i j}\left(x_{1}, x_{2}\right) u\right) \exp \left(-\theta_{i j} u\right), x_{1}, x_{2} \in \mathbb{D}, u \geq 0 .
$$

Thus it suffices to show that for every fixed $u \geq 0, C_{i j}\left(x_{1}, x_{2} ; u\right)$ are direct/cross covariances in $\mathbb{D}$. 
Since $g_{i j}\left(x_{1}, x_{2}\right)$ is a non-negative function, $\exp \left(-g_{i j}\left(x_{1}, x_{2}\right) u\right), x_{1}, x_{2} \in \mathbb{D}$, is a covariance function for every fixed $u \geq 0$, by assumption. An $m \times m$ matrix with entries $\exp \left(-\theta_{i j} u\right)$ is positive definite, since $\Theta$ is conditionally negative definite. Hence, by Theorem 4 of Ma (2011c), the $m \times m$ matrix function with entries $C_{i j}\left(x_{1}, x_{2} ; u\right)$ is a covariance matrix function. 


\section{CHAPTER 6}

\section{Concluding Remarks}

In this dissertation, we have developed an appealing way to construct a new vector ran-

dom field named K-differenced vector random field, whose finite-dimensional distributions belong to the family of the light, long, and thin tailed distributions. The constructions of a K-differenced random variable, random vector, and vector random field are taken as the essential foci of this dissertation, and are made step by step as the scale mixture of Gaussian ones.

The mixing random variable $U$ with density (2.1) plays an important role in the construction, and reduces to a Weibull random variable in the extreme case where $\alpha_{1}$ tends to $\alpha_{2}$. It results in the univariate, multivariate, or finite-dimensional Laplace (double exponential) distributions. Hence, one may apply our K-differenced one to where the Laplace one has been used. It would be of interest to compare the results obtained here with those obtained by using another mixing variable $U$.

We performed the maximum likelihood estimation for the four parameters of a univariate or multivariate K-differenced distribution. Although maximum likelihood estimators are often hard to calculate, they can be estimated numerically by using an optimization method, such as the Nelder-Mead method, and using an advanced computer software. As we showed in Chapters 2 and 3, the maximum likelihood estimation is favorable. This would help us to use the K-differenced distribution to fit a real data set.

For the K-differenced random vector the simulation is not straightforward like the Kdifferenced random variable. We generated the data by implementing three steps: first generate data from the nonnegative random variable $U$ by using the rejection-acceptance 
method, generate data from the normal random vector $Z_{0}$, and then return $U \cdot Z_{0}$ with data to form the K-differenced data. Such a procedure may be extended to simulate the sample from a K-differenced vector random field.

Chapter 4 defines a K-differenced vector random field as a scale mixture of Gaussian vector random fields, which is actually an elliptically contoured vector random field. Its finite-dimensional characteristic functions are derived, and some properties are presented. As one of its most valuable properties, a K-differenced vector random field is characterized by its mean and covariance matrix functions just like a Gaussian one.

In the last chapter we introduced some types of K-differenced covariance matrix structures. They are divided into two groups in Sections 5.2 and 5.3 due to the construction methods. Covariance matrix structures in Section 5.2 contain three ingredients: a complete monotone function, a scalar variogram, and a conditionally negative definite matrix. Second 5.3 constructs covariance matrix structures via the mixture procedure of Ma (2011b). All covariance matrix structures we have constructed in this dissertation are not only obtainable for the K-differenced vector random field but also suitable for other elliptically contoured vector random fields, such as Gaussian, Student's t, Laplace, and logistic random fields. 
REFERENCES 


\section{LIST OF REFERENCES}

[1] Aberg, S. and Podgorski, K. (2011) A class of non-Gaussian second order random fields. Extremes, 14, 187-222.

[2] Balakrishnan, N., Ma, C. and Wang, R. (2015) Logistic vector random field with logistic direct and cross covariances. Journal of Statistical Planing and Inference, 161, 109-118.

[3] Bartlett, M.S. (1934) The vector representation of a sample. Proc. Camb. Phil. Soc., 30, 327-40.

[4] Bateman,H. (1954) Tables of Integral Transforms. Vol.I. McGraw-Hill, New York.

[5] Bapat,R. and Raghaven, T.E.S. (1997) Non-negative Matrices and Application. Cambridge University Press,Cambridge. UK.

[6] Blake, I.F. and Thomas, I.B. (1968) On a class of processes arising in linear estimation theory. IEEE. Trans. Inf. Theory, 14, 12-16.

[7] Cambanis, S., Huang, S., and Simons, G. (1981) On the theory of elliptically contoured distributions. Journal of Multivariate Analysis 11, 368-385.

[8] Chamberlain, G. (1983) A characterization of the distributions that imply meanvariance utility Functions. Journal of Economic Theory 29, 185-201.

[9] Chmielewski, M. A. (1981) Elliptically symmetric distribution: A review and bibliography. Inter. Statist. Review, 49, 67-74.

[10] Chiles,J.P. and Delfiner, P. (1999) Geostatistics: Modeling Spatial Uncertainty. Wiley, New York.

[11] Chu, K.C. (1973) Estimation and decision for linear systems with elliptical random processes. IEEE Transactions On Automation Control, AC18, 499-505. 


\section{LIST OF REFERENCES (continued)}

[12] Cressie, N. (1993) Statistics for Spatial Data. Wiley, New York.

[13] Dempster, A.P. (1969) Elements of Continuous Multivariate Analysis. Reading, Mass.: Addison- Wesley.

[14] Du,J. and Ma,C. (2011) Spherically invariant vector random field in space and time. IEEE Trans. Signal Process, 5921-5929.

[15] Du,J. and Ma,C. (2012) Variogram matrix functions for vector random fields with second-order increments. Math. Geosci. 44,411-425.

[16] Fishman, G. S. (1996) Monte Carlo: Concepts, Algorithms, and Applications. Springer Series in Operations Research. Springer-Verlag. New York.

[17] Fang,K.T., Kotz, S., and Ng, K.W. (1990) Symmetric Multivariate and Related Distributions. Chapman and Hall, London.

[18] Hrdle, W. and Simar, L. (2012) Applied Multivariate Statistical Analysis (3rd ed). Springer.

[19] Hartman, P. and Wintner, A. (1940) On the spherical approach to the normal distribution law. Am. J. Math., 62, 759-79.

[20] Goodman, I. R., and Kotz, S. (1981) Hazard rate based on isoprobability contours. Statistical Distributions in Scientific Work, Vol. 5 (eds G. P. Patil et al.) Boston/London: D. Reidel, pp.289-309.

[21] Gradshteyn, I.S. and Ryzhik, I.M. (2007) Tables of Integrals, Series, and Products. 7th ed. Elsevier, Amsterdam.

[22] Karris, T. S. (2004) Numerical Analysis Using MATLAB and Spreadsheets: Second Edition, EBook 


\section{LIST OF REFERENCES (continued)}

[23] Kelker, D. (1971) Infinite divisibility and variance mixtures of the normal distribution. Ann. Math. Statis., 42(2), 802-8.

[24] Kotz, S. (1975) Multivariate Distributions at a Cross-road. In Statistical Distributions in Scientific Work, 1. (eds G.P. Patil. S. Kotz and) J.K. Ord, D. Reidel Publ. Co.

[25] Kotz, S., Kozubowski, T.J. and Podgrski, K. (2001) The Laplace Distribution and Generalizations: A Revisit with Applications to Communications, Economics, Engineering and Finance. Birkhuser, Boston.

[26] Landsman, Z. M. and Valdez, E. A. (2003). Tail conditional expectations for elliptical distributions. North American Actuarial Journal, 7, 55-71.

[27] Ma, C. (2005) Semiparametric spatio-temporal covariance models with autoregressive temporal margin. Annuals of the Institute of Statistical Mathematics 57, 221-233.

[28] Ma, C. (2005) Spatio-temporal variograms and covariance models. Adv. Appl.Prob, 37, $706-725-725$

[29] Ma, C. (2009) Construction of non-Gaussian random field with any given correlation structure. Journal of Statistical Planing an Inference 139, 780-787.

[30] Ma, C. (2011a). Vector random field with second-order moments or second-order increments. Stoch. Anal. Appl. 29, 197-215.

[31] Ma, C. (2011b) Vector random fields with long-rang dependence. Fractal, 19, 249-258.

[32] Ma, C. (2011c). Covariance matrices for second-order vector random fields in space and time. IEEE Trans. Signal Process. 59,2160-2168.

[33] Ma, C. (2011d) Covariance matrix function of vector $\chi^{2}$ random fields in space and time. IEEE Transactions on Communication 59, 2554-2561. 


\section{LIST OF REFERENCES (continued)}

[34] Ma, C. (2013) Student's t vector random fields with power-law and log-law decaying direct and cross covariance. Stochastic Analysis and Application, 31, 167-182.

[35] Ma, C. (2013) Mittag-Leffler vector random fields with Mittage-Leffler direct and cross covariance functions. Annals of the Institute of Statistical Mathematics 65,941-958

[36] Marshall, A.W. and Olkin, I. (1993). Maximum likelihood characterizations of distributions. Statist Sinica 3, 157-171.

[37] Maxwell, J. C. (1860) Illustration of the dynamical theory of gases. Part I. On the motions and collisions of perfectly elastic bodies. Phil. Mag., 19, 19-32.

[38] McGraw, D.K. and Wagner, J.F. (1968) Elliptically symmetric distributions. IEEE Trans. Inf. Theory, IT14, 110-20.

[39] Mukhopadhyay, P (2011) An Introduction to the Theory of Probability. World Scientific Publishing Company.

[40] Nelder, J. A. and Mead, R. (1965) A simplex method for function minimization. Computer Journal, 7, 308-313.

[41] Osiewalski, J. and Steel, M.F.J. (1993) Robust Bayesian inference in LQ-spherical models. Biometrika 80, 456-460.

[42] Pollard, H. (1946) The representation of $\exp \left(-x^{\lambda}\right)$ as a Laplace integral. Bullerin of the American Mathematical Society 52, 908.

[43] Podgrski, K. and Wegener, J. (2011) Estimation for stochastic models driven by Laplace motion,. Comm. Statist. Theory Methods 18, 3281-3302.

[44] Resnick, S.I. (2006). Heavy-tail Phenomena: Probabilistic and Statistics Modeling. Springer, New York. 


\section{LIST OF REFERENCES (continued)}

[45] Schilling, R.L., Song,R., and Vondracek, Z. (2010) Bernstein Functions: Theory and Applications. Berlin, Germany: De Gruyter.

[46] Sainudiin, R. and Lee, D. (2011) Computational Statistical Experiments in Matlab. Department of Mathematics and Statistics, University of Canterbury, Christchurch, New Zealand

[47] Samorodnitsky,G. and Taqqu,M. S., (1994) Stable non-Gaussian Random Processes : Stochastic Models with Infinite Variance. Chapman and Hall/CRC.

[48] Yaglom,A.M. (1987) Correlation Theory of Stationary and Related Random Function, Vol. I: Basic Results. Spring-Verlag, New York. 
APPENDICES 


\section{APPENDIX A}

\section{MatLab code used in the $\mathrm{K}$-differenced random variable}

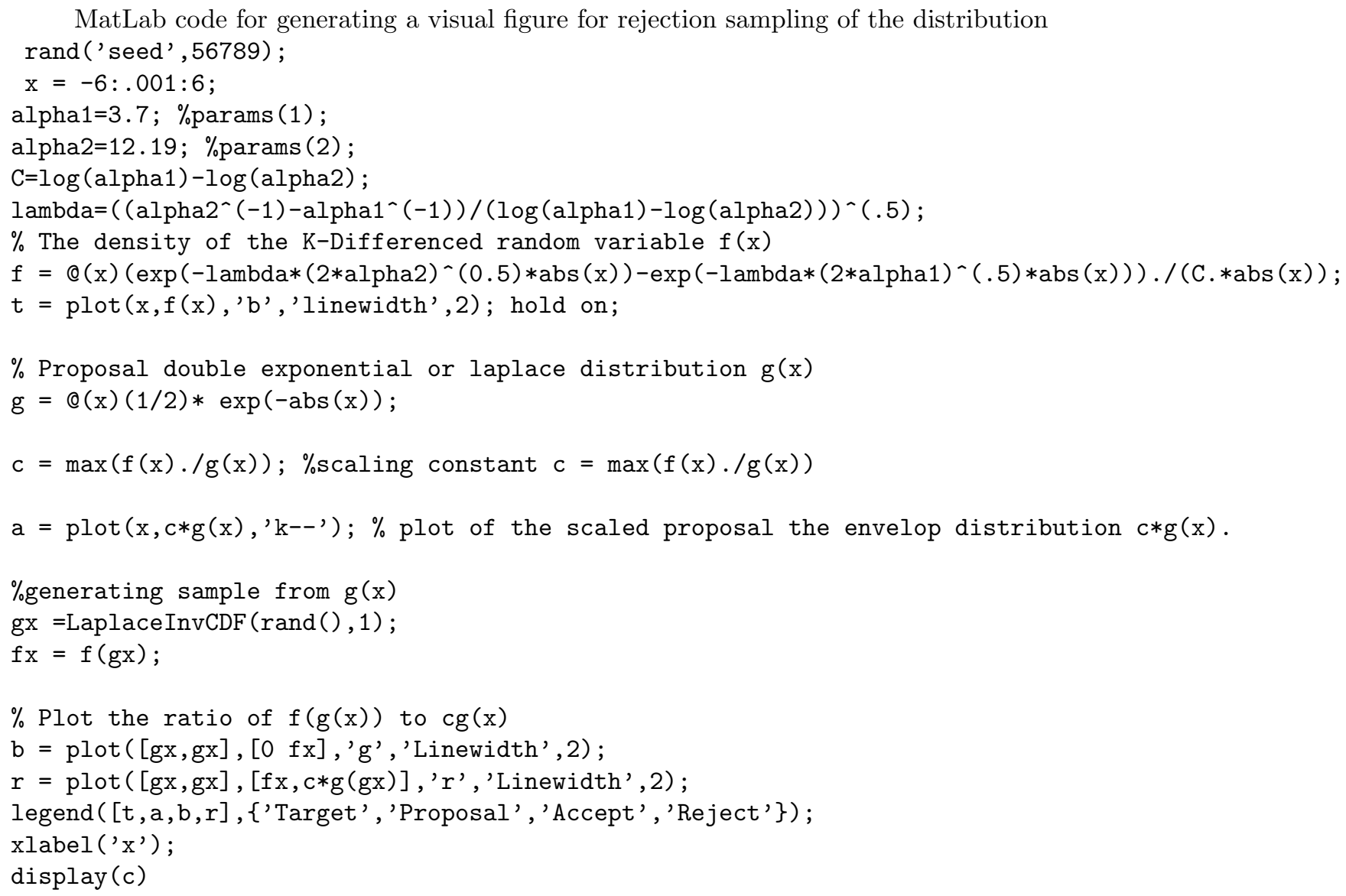

The following is an m.file to generate random number from the K-differenced random variable.

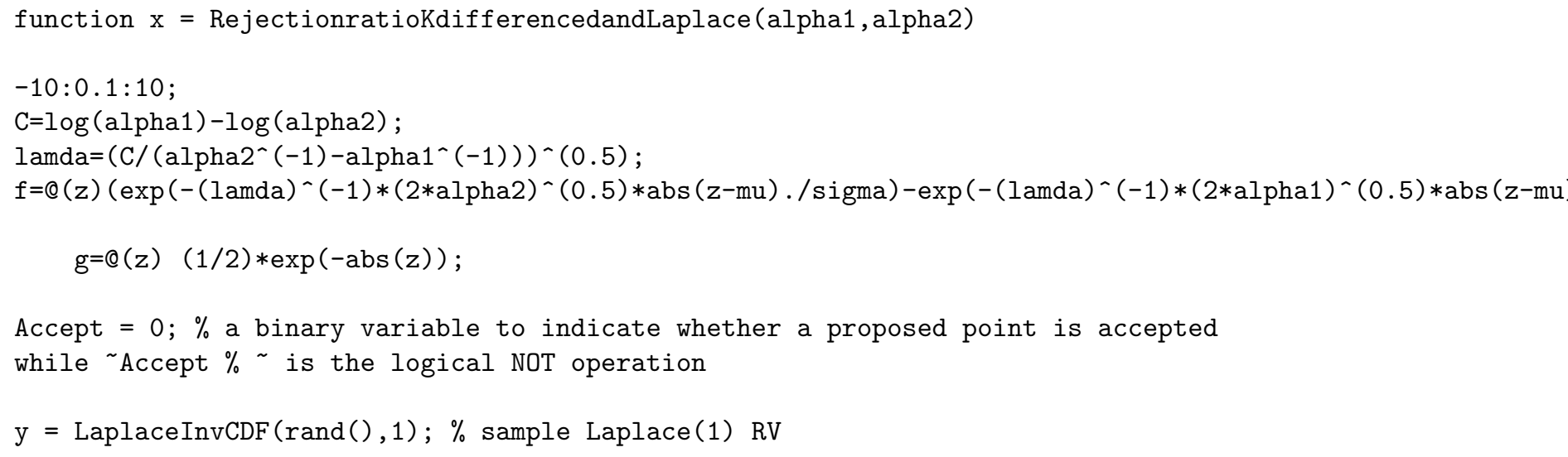




\section{APPENDIX A (continued)}

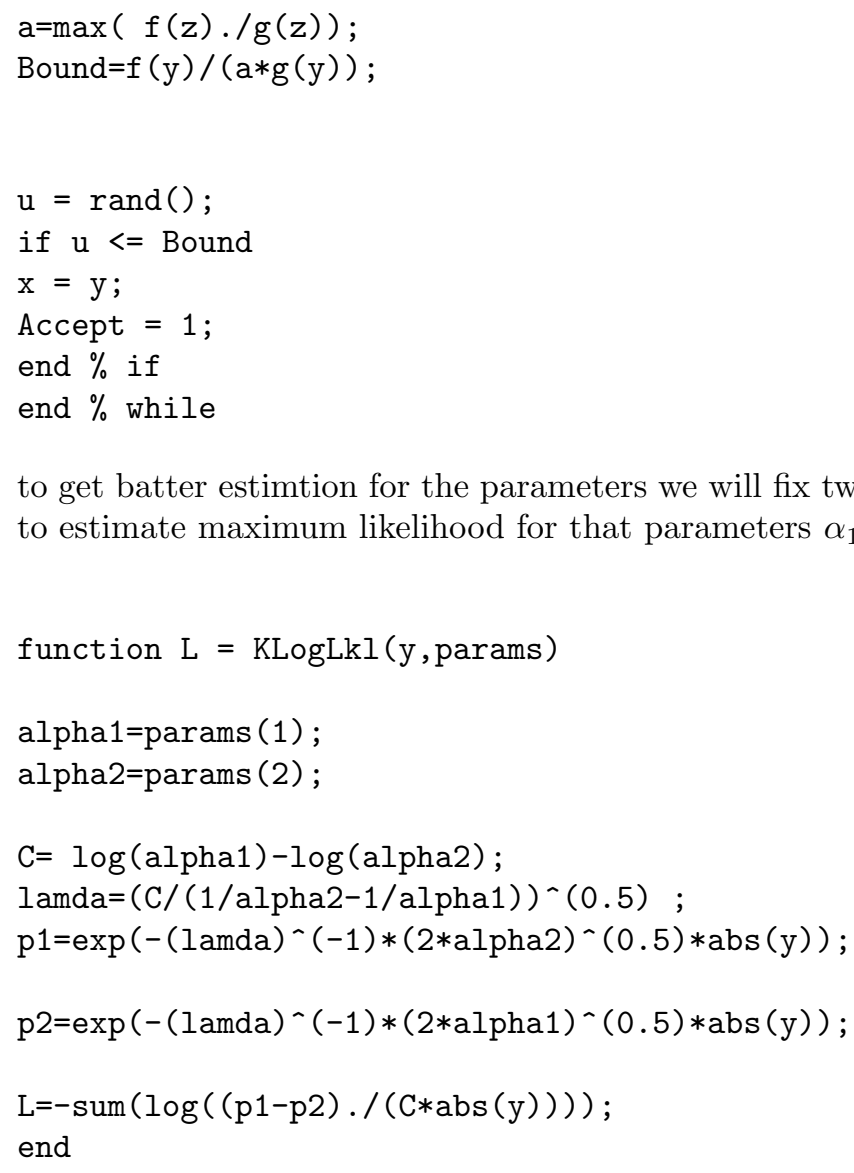




\section{APPENDIX A (continued)}

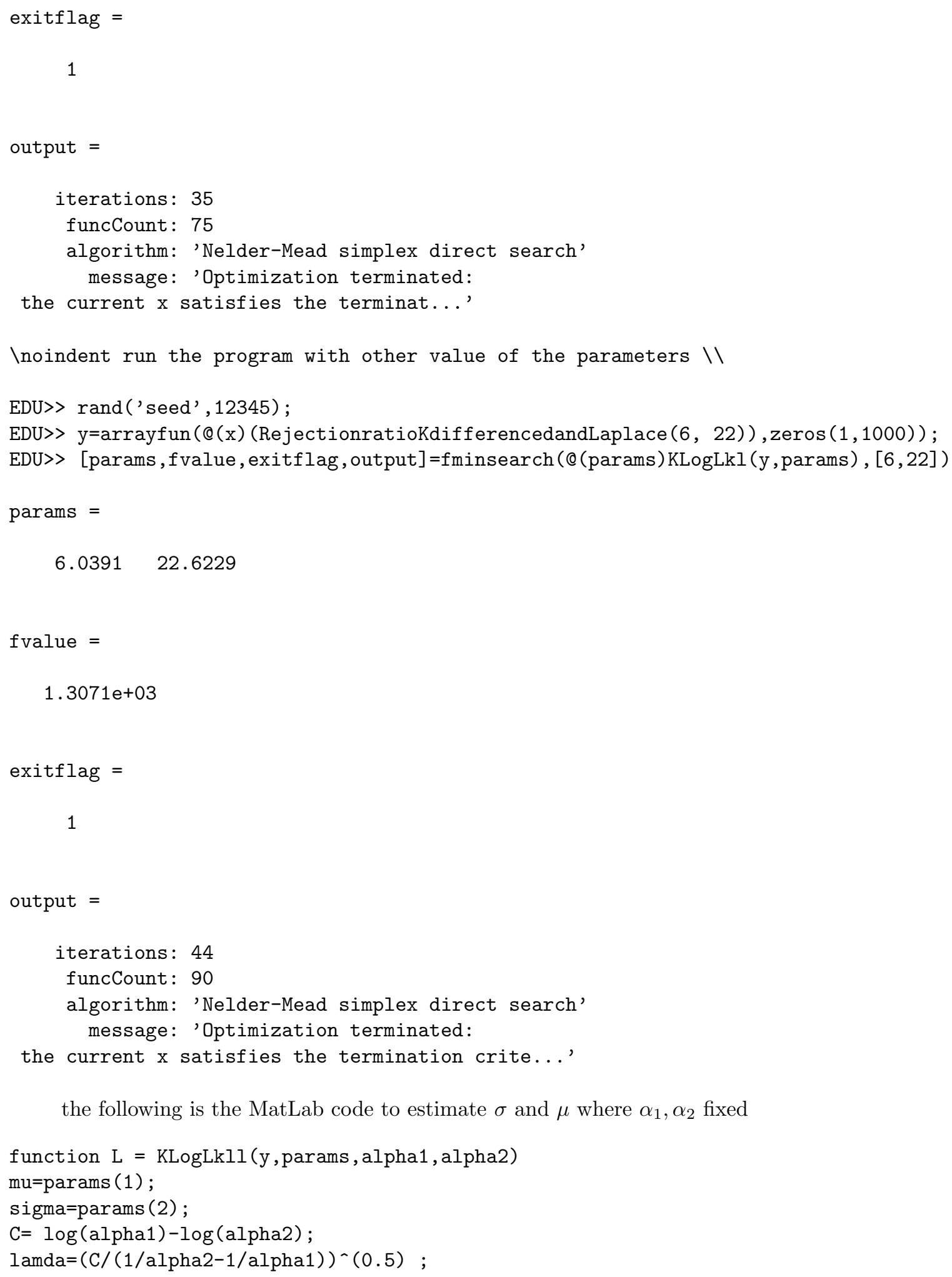




\section{APPENDIX A (continued)}

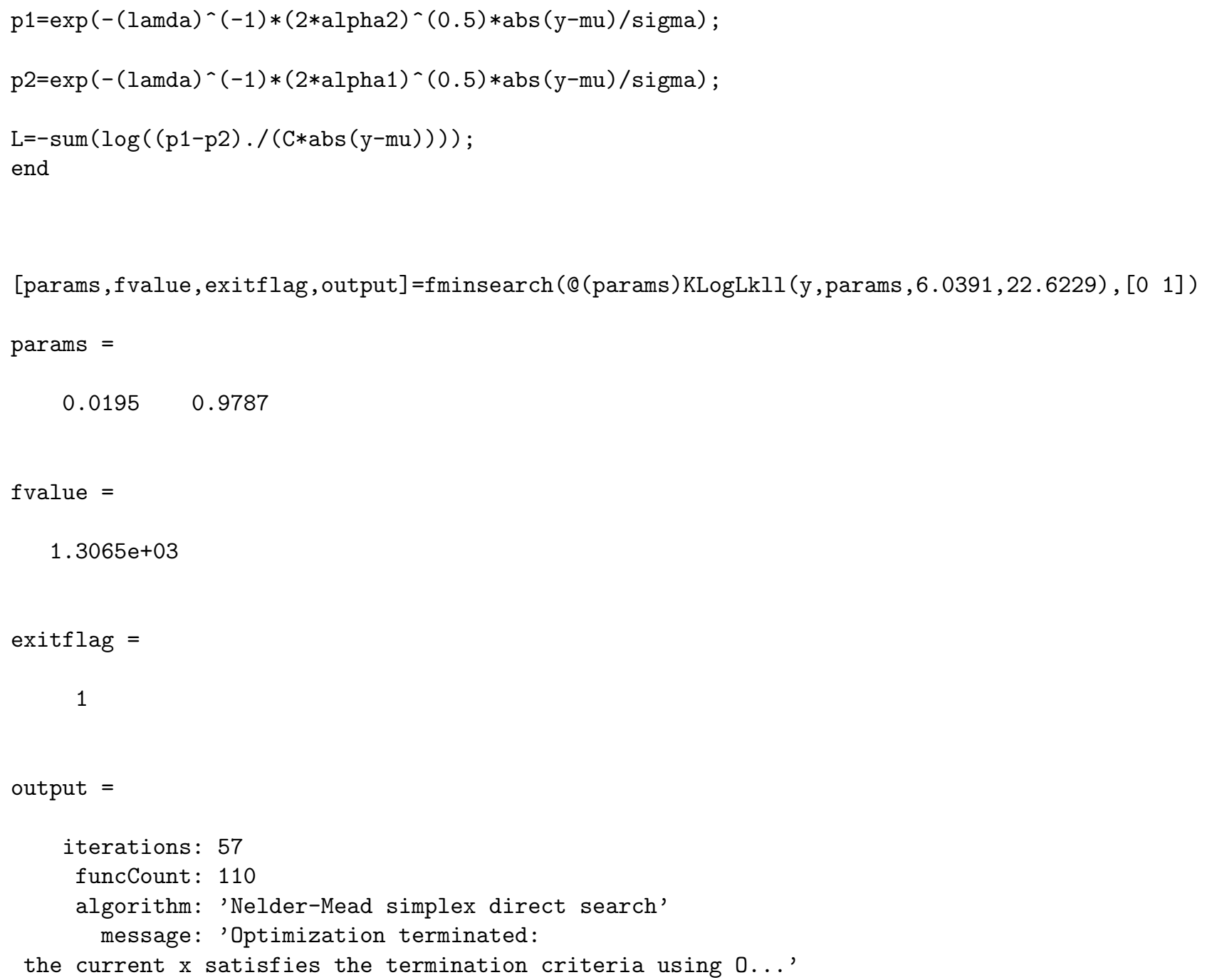


APPENDIX A (continued)

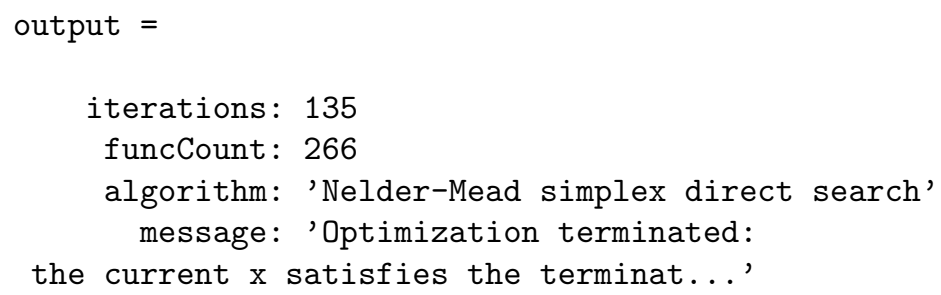




\section{APPENDIX B}

\section{MatLab code used in the $\mathrm{K}$-differenced random vector}

the MatLab script of visual Rejection Sampling from nonnegative random variable $U$ with density $f_{U}(u)$ based on proposal from $\operatorname{Normal}(0,1)$ with PDF g.

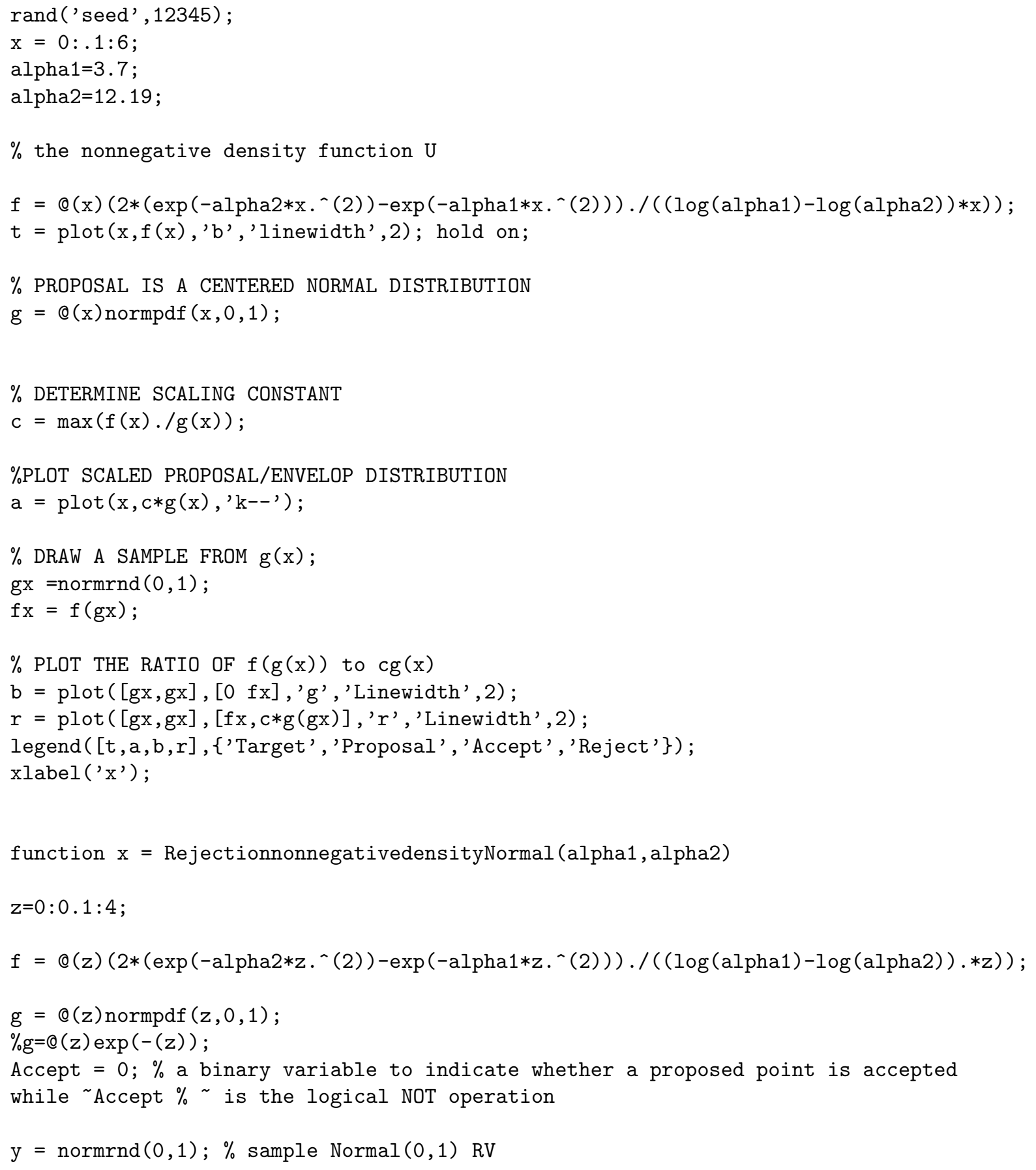




\section{APPENDIX B (continued)}

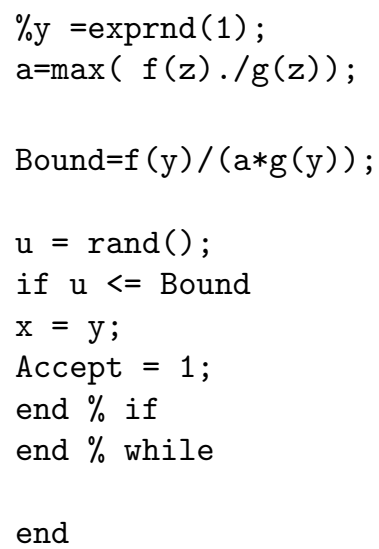

we are going to estimate the parameters by fixing or giving the true value of $\alpha_{1}$ and estimate the $\alpha_{2}$ then do the same thing for estimating $\alpha_{1}$.

function $\mathrm{L}=\mathrm{KLogLk} 2$ (g,params)

$\mathrm{x}=\mathrm{g}(:, 1)$;

$\mathrm{y}=\mathrm{g}(:, 2)$; 


\section{APPENDIX B (continued)}

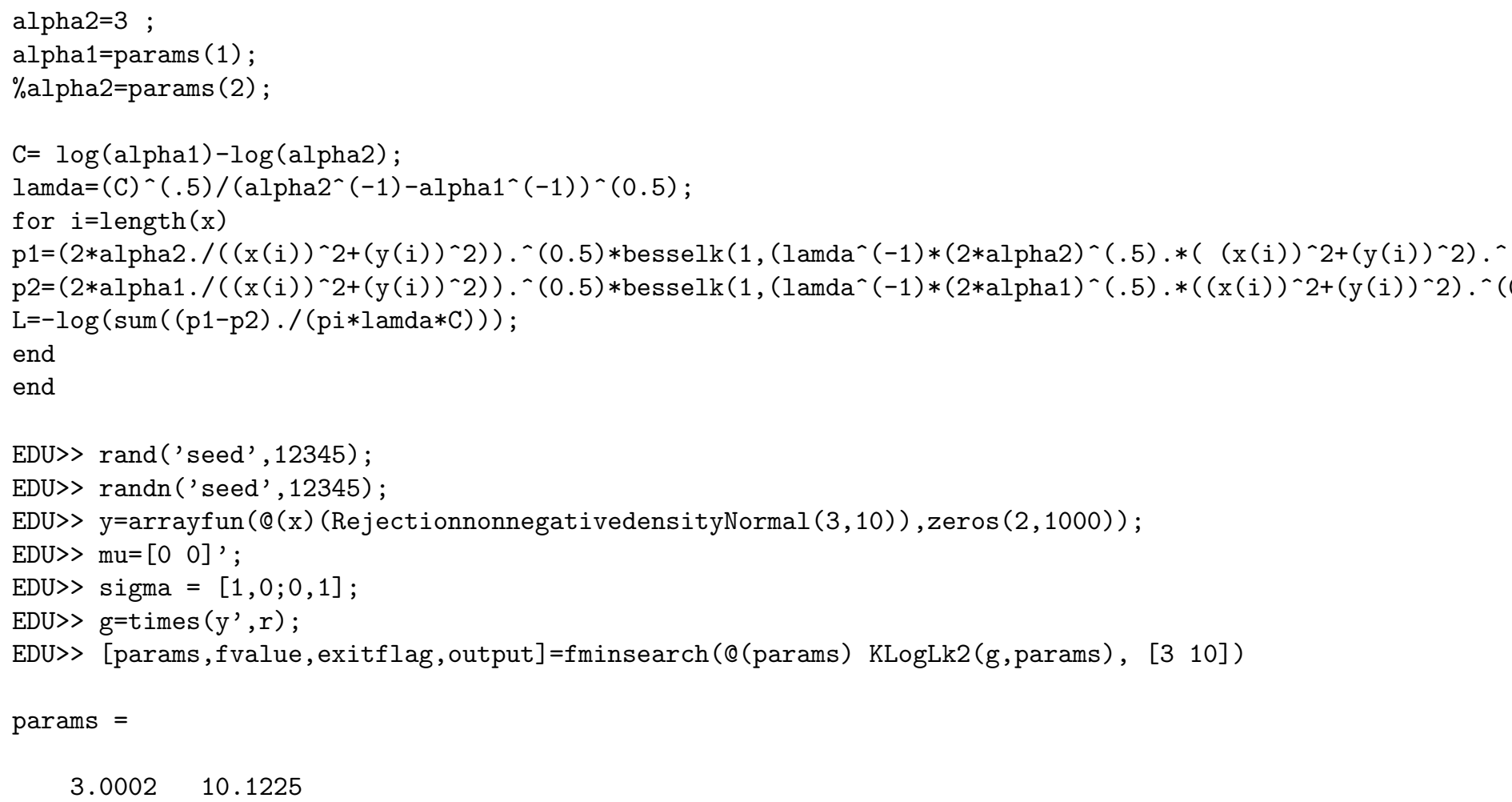




\section{fvalue $=$ \\ 2.3094 \\ exitflag $=$}

1

output $=$

iterations: 44

funcCount: 100

algorithm: 'Nelder-Mead simplex direct search' message: 'Optimization terminated:

the current $\mathrm{x}$ satisfies the termination criteria us...' 